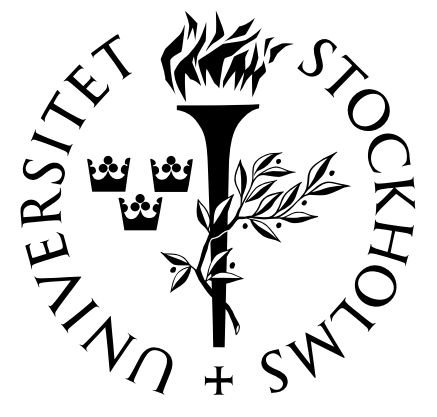

\title{
Differential Operators on Hyperplane Arrangements
}

Pär Holm

Research Reports in Mathematics

Number 3, 2002

Department of Mathematics

STOCKHOLM UNIVERSITY 
Electronic versions of this document are available at http://www.matematik.su.se/reports/2002/3

Date of publication: March 12, 2002

Postal address:

Department of Mathematics

Stockholm University

S-106 91 Stockholm

Sweden

Electronic addresses:

http://www.matematik.su.se

info@matematik.su.se 


\title{
Differential Operators on Hyperplane Arrangements
}

\author{
Pär Holm
}

March 11, 2002

\begin{abstract}
In this paper we show that if $\mathcal{A}$ is a hyperplane arrangement in $k^{n}$, where $k$ is a field of characteristic zero, and if $A$ is the coordinate ring of $\mathcal{A}$, then the ring of $k$-linear differential operators, $\mathcal{D}(A)$, has a nice decomposition, $\mathcal{D}(A)=\bigoplus \mathcal{D}^{(m)}(A)$, as an $A$-module. We also show that if $\mathcal{A}$ is generic, then $\mathcal{D}(A)$ is finitely generated as a $k$-algebra.
\end{abstract}

\section{Introduction}

Throughout this paper, we will let $k$ be a field of characteristic zero, $n$ a positive integer, and $R=k\left[x_{1}, \ldots, x_{n}\right]$.

Let $A$ be a commutative $k$-algebra. We let $\mathcal{D}(A)$ denote the ring of $k$-linear differential operators on $A$. Then $\mathcal{D}(A)$ is an $A$-module in an obvious way, with $\operatorname{Der}(A)$, the derivations on $A$, as an $A$-submodule.

The differential operators, $\mathcal{D}(R)$, on $R$ is the $n$-th Weyl algebra over $k$, i.e., the free associative algebra

$$
k\left\langle x_{1}, \ldots, x_{n}, \partial_{1}, \ldots, \partial_{n}\right\rangle
$$

modulo the relations

$$
x_{i} x_{j}-x_{j} x_{i}=\partial_{i} \partial_{j}-\partial_{j} \partial_{i}=0 \text { and } \partial_{i} x_{j}-x_{j} \partial_{i}=\delta_{i j},
$$

where $\delta_{i j}$ is the Kronecker $\delta$, and where we write $\partial_{i}$ in place of $\frac{\partial}{\partial x_{i}}$.

If $I$ is an ideal in $R$ and $A=R / I$, then

$$
\mathcal{D}(A) \cong \mathcal{D}(I) / I \mathcal{D}(R),
$$

where $\mathcal{D}(I)$ is the subring of $\mathcal{D}(R)$ of operators preserving $I$, and $I \mathcal{D}(R)$ is the ideal of operators with polynomial coefficients in $I$. Thus each element in $\mathcal{D}(A)$ may be written on the form

$$
\sum_{\alpha} \overline{f_{\alpha}} \partial^{\alpha}
$$

where $f_{\alpha} \in R$ and $\overline{f_{\alpha}}$ denotes the image of $f_{\alpha}$ in $A$.

The ring $\mathcal{D}(I)$ is an $R$-submodule of $\mathcal{D}(R)$, and $\operatorname{Der}(I)$, the derivations on $R$ preserving $I$, is an $R$-submodule of $\mathcal{D}(I)$. 
An operator in $\mathcal{D}(R)$ of the form

$$
\sum_{|\alpha|=m} f_{\alpha} \partial^{\alpha}
$$

will be said to be of homogeneous order $m$, as will the operator in $\mathcal{D}(A)$, induced by (2).

Let $\mathcal{D}^{(m)}(I)$ and $\mathcal{D}^{(m)}(R)$ be the $R$-submodules of $\mathcal{D}(I)$ and $\mathcal{D}(R)$, respectively, of operators of homogeneous order $m$. Then the $A$-submodule of $\mathcal{D}(A)$ of operators of homogeneous order $m$ will be $\mathcal{D}^{(m)}(A) \cong \mathcal{D}^{(m)}(I) / I^{(m)}(R)$.

Suppose $A=R / I$ is the coordinate ring of a hyperplane arrangement, $\mathcal{A}$, in $k^{n}$. The aim of this paper is to show that $\mathcal{D}(A)$ has a decomposition,

$$
\mathcal{D}(A)=\bigoplus_{m \geq 0} \mathcal{D}^{(m)}(A),
$$

as an $A$-module, and to find explicit, finitely many, $k$-algebra generators for $\mathcal{D}(A)$, if $\mathcal{A}$ is a generic hyperplane arrangement.

This will be done by first giving the corresponding results for $\mathcal{D}(I)$. From this, and (1), we immediately get our results for $\mathcal{D}(A)$.

Even more may be said about the structure of $\mathcal{D}(A)$. Namely, it follows from a result by Másson, [2, Theorem 2.4], that $\mathcal{D}(A)$ is simple if and only if $\mathcal{A}$ only consists of one hyperplane. In this case $A$ is regular, and much is known about the structure of $\mathcal{D}(A)$.

Let us now outline the contents of this paper.

In Section 2.1 we will define $\mathcal{D}(A)$ for an arbitrary commutative $k$-algebra $A$, and state a few of its properties. Induced operators, and the isomorphism (1) are discussed in Section 2.2.

In Section 3 we show some general results on differential operators preserving ideals in $R$, which will be used later on. In particular we prove that

$$
\mathcal{D}\left(\left\langle f_{1} \cdots f_{r}\right\rangle\right)=\bigcap_{i=1}^{r} \mathcal{D}\left(\left\langle f_{i}\right\rangle\right),
$$

if $f_{1}, \ldots, f_{r} \in R$ are nonconstant and pairwise relatively prime.

Hyperplane arrangements are introduced in Section 4. Our general reference for hyperplane arrangements is [4]. An arrangement of hyperplanes passing through the origin is called a central hyperplane arrangement. When we want to emphasize that all hyperplanes in an arrangement $\mathcal{A}$ need not pass through the origin, we call $\mathcal{A}$ an affine hyperplane arrangement.

A central hyperplane arrangement $\mathcal{A}$ in $k^{n}$ is called generic if every intersection of less that $n$ hyperplanes in $\mathcal{A}$ have codimension equal to the number of hyperplanes in the intersection.

Section 5 deals with differential operators on hyperplane arrangements. The first part, Section 5.1, deals with affine hyperplane arrangements. We prove that if $I \subseteq R$ defines an affine hyperplane arrangement, then

$$
\mathcal{D}(I)=\bigoplus_{m \geq 0} \mathcal{D}^{(m)}(I)
$$


as an $R$-module. Hence we have

$$
\mathcal{D}(A)=\bigoplus_{m \geq 0} \mathcal{D}^{(m)}(A)
$$

as an $A$-module, if $A$ is the coordinate ring of an affine hyperplane arrangement.

Section 5.2 deals only with central hyperplane arrangements. We introduce, for each $m \geq 0$, an operator $\varepsilon_{m} \in \mathcal{D}(R)$ of homogeneous order $m$, which preserves any homogeneous ideal in $R$. Then we show, in Section 5.2.1, how to associate to an ideal $I$ defining a generic hyperplane arrangement, a subset

$$
D=\left\{P_{1} \delta_{1}, \ldots, P_{s} \delta_{s}\right\}
$$

of $\operatorname{Der}(I)$, where each $\delta_{i}$ is a derivation with coefficients in $k$, and each $P_{i} \in R$. With this set $D$ at hand, we have

$$
\mathcal{D}^{(m)}(I)=\sum_{|\alpha|=m}\left(I:\left(I: P^{\alpha}\right)\right) \delta^{\alpha}+R \varepsilon_{m},
$$

as an $R$-module, for each $m \geq 0$.

The proof of this uses a method known as deletion and restriction. Given a central hyperplane arrangement $\mathcal{A}$, there is a deleted, $\mathcal{A}^{\prime}$, and a restricted, $\mathcal{A}^{\prime \prime}$, hyperplane arrangement. If $I, I^{\prime}$, and $I^{\prime \prime}$ are the defining ideals for these hyperplane arrangements, there is (compare [4, Proposition 4.45]) an exact sequence of the form

$$
0 \longrightarrow \operatorname{Der}\left(I^{\prime}\right) \longrightarrow \operatorname{Der}(I) \longrightarrow \operatorname{Der}\left(I^{\prime \prime}\right) \text {. }
$$

This was used in [8], where a result (Corollary 3.2) which amounts to (4) was given for the case $m=1$, i.e., for $\operatorname{Der}(I)$, although without giving a finite set of generators for $\operatorname{Der}(I)$.

We will generalize the sequence (5) to operators of higher homogeneous order, and then follow the ideas used in [8] to prove (4)

Then we show that $\varepsilon_{m}$ is not needed as a generator if the number of hyperplanes is less than or equal to $n$.

Combining this with (3) and (4) we reach the main result of this section:

If $I$ defines a generic hyperplane arrangement, then

$$
\mathcal{D}(I)=\bigoplus_{m \geq 0}\left(\sum_{|\alpha|=m}\left(I:\left(I: P^{\alpha}\right)\right) \delta^{\alpha}+R \varepsilon_{m}\right),
$$

as an $R$-module, where $R \varepsilon_{m} \subseteq \sum_{|\alpha|=m}\left(I:\left(I: P^{\alpha}\right)\right) \delta^{\alpha}$, if the number of hyperplanes is less than or equal to $n$.

We end Section 5.2.1 with an example showing that (6) is not true in general if the hyperplane arrangement defined by $I$ is not generic.

In Section 5.2.2 finally, we will use (6) to show that if $I$ defines a generic hyperplane arrangement, then $\mathcal{D}(I)$, and hence $\mathcal{D}(A)$ if $A=R / I$, is finitely generated as a $k$-algebra. More precisely, for each multi-index $\alpha$, the ideal $\left(I:\left(I: P^{\alpha}\right)\right)$ is principal. For each such ideal we choose a generator, $P_{\alpha}$, and then we show that only finitely many of the operators $P_{\alpha} \delta^{\alpha}$, together with $x_{1}, \ldots, x_{n}$ and $\varepsilon=\varepsilon_{1}$ are needed to generate $\mathcal{D}(I)$ as a $k$-algebra. 
Acknowledgement. The author would like to express his appreciation to his former advisor, Gísli Másson, for introducing him to the subject of rings of differential operators, and for proposing the study of operators on central hyperplane arrangements. The author is also grateful to his present advisors, Ralf Fröberg and Clas Löfwall, for their guidance and support during the work on this paper.

\section{Differential Operators}

We begin, in Section 2.1, with the definition of the ring of differential operators on a commutative $k$-algebra, and give some of its properties. Then, in Section 2.2, we will see what the differential operators on a quotient of a polynomial algebra looks like.

\subsection{The Ring of Differential Operators}

Let $A$ be a commutative $k$-algebra. Every element $a \in A$ defines a $k$-linear endomorphism $\theta_{a}$ of $A$ by $\theta_{a}(b)=a b$, for $b \in A$. If $\varphi \in \operatorname{End}_{k}(A)$, we will write $a \varphi$ and $\varphi a$ for $\theta_{a} \varphi$ and $\varphi \theta_{a}$, respectively. With this notation, letting $A$ operate by left multiplication gives $\operatorname{End}_{k}(A)$ an $A$-module structure. As usual, $[\varphi, \psi]$ denotes the commutator $\varphi \psi-\psi \varphi$.

Definition 2.1. Set

$$
\mathcal{D}_{k}^{0}(A)=\left\{\theta \in \operatorname{End}_{k}(A) \mid[\theta, a]=0 \text { for all } a \in A\right\}=\operatorname{End}_{A}(A)=A,
$$

by identifying $a \in A$ with $\theta_{a} \in \operatorname{End}_{k}(A)$. For $m \geq 1$, define

$$
\mathcal{D}_{k}^{m}(A)=\left\{\theta \in \operatorname{End}_{k}(A) \mid[\theta, a] \in \mathcal{D}_{k}^{m-1}(A) \text { for all } a \in A\right\} .
$$

The ring of differential operators on $A$ is now defined as

$$
\mathcal{D}_{k}(A)=\bigcup_{m \geq 0} \mathcal{D}_{k}^{m}(A) .
$$

The order of an element $\theta \in \mathcal{D}_{k}(A)$ is the least $m$ such that $\theta \in \mathcal{D}_{k}^{m}(A)$.

This definition gives $\mathcal{D}_{k}(A)$ the structure of a filtered ring since, by induction,

$$
\mathcal{D}_{k}^{m}(A) \mathcal{D}_{k}^{l}(A) \subseteq \mathcal{D}_{k}^{m+l}(A) \text { and } \mathcal{D}_{k}^{m}(A) \subseteq \mathcal{D}_{k}^{m+1}(A),
$$

for all $m$ and $l$. Each $\mathcal{D}_{k}^{m}(A)$, and thereby also $\mathcal{D}_{k}(A)$, is an $A$-submodule of $\operatorname{End}_{k}(A)$.

The derivations on $A$ are defined as

$$
\operatorname{Der}_{k}(A)=\left\{\theta \in \operatorname{End}_{k}(A) \mid \theta(a b)=\theta(a) b+a \theta(b) \text { for all } a, b \in A\right\} .
$$

This too is an $A$-submodule of $\operatorname{End}_{k}(A)$.

Since there is no risk for confusion we will from now on suppress the reference to the field $k$ in the notation.

The $k$-subalgebra of $\operatorname{End}(A)$ generated by $A$ and $\operatorname{Der}(A)$ will be denoted by $\Delta(A)$. This too has the structure of a filtered ring, by letting $\Delta^{m}(A)$ be the $A$-submodule generated by all products of at most $m$ derivations in $\operatorname{Der}(A)$.

The results in the following lemma can all be found in [3]. 


\section{Lemma 2.2.}

(i) $\mathcal{D}^{1}(A)=A \oplus \operatorname{Der}(A)$.

(ii) $\Delta(A)$ is a filtered subring of $\mathcal{D}(A)$.

(iii) If $A$ is regular, then $\mathcal{D}(A)=\Delta(A)$, and their filtrations coincide.

(iv) $\operatorname{Der}(R)$ is a free $R$-module of rank $n$, generated by $\left\{\frac{\partial}{\partial x_{1}}, \ldots, \frac{\partial}{\partial x_{n}}\right\}$.

The converse of (iii), that $A$ is regular if $\mathcal{D}(A)=\Delta(A)$, in the case that $A$ is finitely generated as a $k$-algebra is a well known conjecture by Nakai. It has been shown, in [6], that Nakai's conjecture holds when $A$ is the coordinate ring of a reduced affine variety, all of whose irreducible components are nonsingular.

Part (iii) and (iv) in the lemma above shows that $\mathcal{D}(R)$ is the $n$-th Weyl algebra over $k$. For more details on the Weyl algebra, we refer to [1].

Henceforth, we will write $\partial_{i}$ for $\frac{\partial}{\partial x_{i}}$. We will also use multi-indices, i.e.,

$$
x^{\alpha}=x_{1}^{\alpha_{1}} \cdots x_{n}^{\alpha_{n}} \text { and } \partial^{\beta}=\partial_{1}^{\beta_{1}} \cdots \partial_{n}^{\beta_{n}}
$$

if

$$
\alpha=\left(\alpha_{1}, \ldots, \alpha_{n}\right) \text { and } \beta=\left(\beta_{1}, \ldots, \beta_{n}\right) .
$$

The multi-index with 1 in position $i$ and zeros elsewhere will be denoted by $e_{i}$. To further simplify notation, we will write

$$
|\alpha|=\alpha_{1}+\ldots+\alpha_{n}
$$

and

$$
\alpha !=\alpha_{1} ! \cdots \alpha_{n} !
$$

An operator in $\mathcal{D}(R)$ of the form

$$
\theta=x^{\alpha} \partial^{\beta}
$$

will be called a monomial.

We will let $*$ denote the left $\mathcal{D}(A)$-module operation on $A$.

The following proposition appears as Proposition 1.2 in chapter one of [1].

Proposition 2.3. Any operator $\theta \in \mathcal{D}(R)$ may be uniquely written on the form

$$
\theta=\sum c_{\alpha, \beta} x^{\alpha} \partial^{\beta},
$$

where $c_{\alpha, \beta} \in k$. That is, the monomials $\left\{x^{\alpha} \partial^{\beta}\right\}$ is a basis for $\mathcal{D}(R)$ as a vector space over $k$.

It is easy to see that an operator $\theta \in \mathcal{D}(R)$ is completely determined by its action on the monomials $x^{\alpha}$, with $|\alpha| \leq$ order of $\theta$. We formulate this as a lemma, whose proof will be omitted.

Lemma 2.4. Let $\theta$ and $\psi$ be operators of order $m$ in $\mathcal{D}(R)$. If

$$
\theta * x^{\alpha}=\psi * x^{\alpha},
$$

for all $\alpha$ with $|\alpha| \leq m$, then $\theta=\psi$. 


\subsection{Induced Operators}

The results in this section may all be found in [3].

Let $A=R / I$, where $I$ is an ideal in $R$. For $f \in R$, we let $\bar{f}$ denote the image of $f$ in $A$ under the canonical projection.

An element $\theta \in \operatorname{End}(R)$ induces a well defined element $\bar{\theta} \in \operatorname{End}(A)$ by

$$
\bar{\theta}(\bar{f})=\overline{\theta(f)}
$$

if and only if $\theta(I) \subseteq I$. In this case, $\bar{\theta}=0$ if and only if $\theta(R) \subseteq I$.

Furthermore, if $\theta \in \mathcal{D}(R)$ and $\theta * I \subseteq I$, then $\bar{\theta} \in \mathcal{D}(A)$, and the order of $\bar{\theta}$ is less than or equal to the order of $\theta$. Let

$$
\mathcal{D}(I)=\{\theta \in \mathcal{D}(R) \mid \theta * I \subseteq I\}
$$

and

$$
I \mathcal{D}(R)=\left\{\sum f_{\alpha} \partial^{\alpha} \in \mathcal{D}(R) \mid f_{\alpha} \in I \text { for all } f_{\alpha}\right\} .
$$

Then, if $\theta \in \mathcal{D}(R)$, we have $\theta * R \subseteq I$ if and only if $\theta \in I \mathcal{D}(R)$. It is clear that $I \mathcal{D}(R)$ is a right ideal in $\mathcal{D}(R)$, but it is also true that $\mathcal{D}(I)$ is the largest subring of $\mathcal{D}(R)$ in which $I \mathcal{D}(R)$ is a twosided ideal.

We may now formulate the following important result.

Theorem 2.5. Let $A=R / I$. Then

$$
\mathcal{D}(A) \cong \mathcal{D}(I) / I \mathcal{D}(R),
$$

and this isomorphism preserves the filtrations.

Thus any element in $\mathcal{D}(A)$ may be written as

$$
\sum_{\alpha} \overline{f_{\alpha}} \partial^{\alpha}
$$

where $\sum f_{\alpha} \partial^{\alpha} \in \mathcal{D}(R)$, and where we mean that

$$
\left(\sum_{\alpha} \overline{f_{\alpha}} \partial^{\alpha}\right) * \bar{g}=\sum_{\alpha} \overline{f_{\alpha} \partial^{\alpha} * g}
$$

for all $\bar{g} \in A$.

\section{General Results on Operators Preserving Ideals in $R$}

The results in this section will be used in Section 5 .

Suppose that $I=\left\langle f_{1}, \ldots, f_{r}\right\rangle$ is an ideal in $R$, and that $\theta \in \mathcal{D}(R)$. Then $\theta$ belongs to $\mathcal{D}(I)$ if and only if $\theta * x^{\alpha} f_{j} \in I$, for all $\alpha$ and $j=1, \ldots, r$.

With Lemma 2.4 in mind we would expect that $\theta * x^{\alpha} f_{j} \in I$ for a finite number of the $x^{\alpha}$ should suffice to show that $\theta \in \mathcal{D}(I)$. This is indeed so, but to prove this we first need the following lemma. 
Lemma 3.1. Let $I=\left\langle f_{1}, \ldots, f_{r}\right\rangle$ be an ideal in $R$, and let $\theta \in \mathcal{D}(R)$. Then $\theta \in \mathcal{D}(I)$ if and only if

(i) $\left[\theta, x_{i}\right] * I \subseteq I$ for $i=1, \ldots, n$, and

(ii) $\theta * f_{j} \in I$ for $j=1, \ldots, r$.

Proof. This is Proposition 2.10 (i) and (ii) in [5], from which we have taken the proof.

It is obvious that (i) and (ii) holds if $\theta \in \mathcal{D}(I)$. To prove the converse, assume $\theta \in \mathcal{D}(R)$ is such that (i) and (ii) holds. We must show that $\theta * x^{\alpha} f_{j} \in I$ for all $\alpha$ and for $j=1, \ldots, r$. This is done by induction on $|\alpha|$.

The case $|\alpha|=0$ is just assumption (ii) above, so assume that $\theta * x^{\alpha} f_{j} \in I$ for $|\alpha|=m$, and let $\beta=\alpha+e_{i}$, for some $i \in\{1, \ldots, n\}$. Then

$$
\theta * x^{\beta} f_{j}=\theta * x_{i} x^{\alpha} f_{j}=\left[\theta, x_{i}\right] * x^{\alpha} f_{j}+x_{i} \theta * x^{\alpha} f_{j} \in I,
$$

since $\left[\theta, x_{i}\right] * x^{\alpha} f_{j} \in I$ by $(i)$, and $x_{i} \theta * x^{\alpha} f_{j} \in I$ by the induction hypothesis.

Proposition 3.2. Let $I=\left\langle f_{1}, \ldots, f_{r}\right\rangle$ be an ideal in $R$, and let $\theta \in \mathcal{D}(R)$ be an operator of order $m \geq 1$. Then $\theta \in \mathcal{D}(I)$ if and only if $\theta * x^{\alpha} f_{j} \in I$ for $|\alpha| \leq m-1$ and $j=1, \ldots, r$.

Proof. It is clear that $\theta * x^{\alpha} f_{j} \in I$ if $\theta \in \mathcal{D}(I)$, so it remains to prove that $\theta \in \mathcal{D}(I)$ if $\theta * x^{\alpha} f_{j} \in I$ for $|\alpha| \leq m-1$ and $j=1, \ldots, r$. To do so, we first observe that any element in $R$ preserves the ideal $I$, so we may without loss of generality assume that the order zero component of $\theta$ is zero. We will now proceed by induction on $m$. If $m=1$, then $\theta \in \operatorname{Der}(R)$ and the assumption is that $\theta * f_{j} \in I, j=1, \ldots, r$. Hence

$$
\theta * h f_{j}=(\theta * h) f_{j}+h \theta * f_{j} \in I,
$$

for every $h \in R$, so $\theta * I \subseteq I$.

Now assume that the proposition is true for some $m \geq 1$ and that $\theta \in \mathcal{D}(R)$ is of order $m+1$ and such that $\theta * x^{\alpha} f_{j} \in I$ for $|\alpha| \leq m$ and $j=1, \ldots, r$. Then $\alpha=\mathbf{0}$ shows $\theta * f_{j} \in I, j=1, \ldots, r$. For any $\beta$ with $|\beta| \leq m-1$ we have $\left|\beta+e_{i}\right| \leq m, i=1, \ldots, n$, so

$$
I \ni \theta * x^{\beta+e_{i}} f_{j}=\theta * x_{i} x^{\beta} f_{j}=\left[\theta, x_{i}\right] * x^{\beta} f_{j}+x_{i} \theta * x^{\beta} f_{j},
$$

which shows that $\left[\theta, x_{i}\right] * x^{\beta} f_{j} \in I$ for $i=1, \ldots, n,|\beta| \leq m-1$, and $j=1, \ldots, r$. But $\left[\theta, x_{i}\right]$ is an operator of order $m$, so $\left[\theta, x_{i}\right] * I \subseteq I, i=1, \ldots, n$, by the induction hypothesis. Thus $\theta * I \subseteq I$ by lemma 3.1.

Lemma 3.3. Let $\left\{I_{j}\right\}_{j \in J}$ be any family of ideals in $R$, and let $I=\bigcap_{j \in J} I_{j}$. Then

$$
\bigcap_{j \in J} \mathcal{D}\left(I_{j}\right) \subseteq \mathcal{D}(I)
$$

Proof. Suppose $\theta \in \bigcap \mathcal{D}\left(I_{j}\right)$. For every $j \in J$ we have $I \subseteq I_{j}$, so $\theta * I \subseteq I_{j}$. It follows that $\theta * I \subseteq I$.

The converse of Lemma 3.3 is not true in general, as the following example shows. 
Example 3.4. Let $I_{1}=\langle x\rangle$ and $I_{2}=\left\langle x^{2}, x y, y^{2}\right\rangle$ be ideals in $k[x, y]$. Then $I=I_{1} \cap I_{2}=\left\langle x^{2}, x y\right\rangle$. By Proposition 3.2, the operator $x \partial_{x} \partial_{y}-\partial_{y}$ preserves $I$, but

$$
\left(x \partial_{x} \partial_{y}-\partial_{y}\right) * y^{2}=-2 y \notin\left\langle x^{2}, x y, y^{2}\right\rangle,
$$

so it does not belong to $\mathcal{D}\left(I_{1}\right) \cap \mathcal{D}\left(I_{2}\right)$.

There are cases in which the converse of Lemma 3.3 is valid, as the next result shows. It concerns the ideal defining a union of (possibly nonreduced) hypersurfaces. It is well known for the special case of derivations, see e.g. Proposition 4.8 in [4].

Theorem 3.5. Suppose $f_{1}, \ldots, f_{r} \in R$ are nonconstant and pairwise relatively prime. Then

$$
\mathcal{D}\left(\left\langle f_{1} \cdots f_{r}\right\rangle\right)=\bigcap_{i=1}^{r} \mathcal{D}\left(\left\langle f_{i}\right\rangle\right) .
$$

Proof. If $r=1$ there is nothing to prove, so we may assume that $r>1$. Since the $f_{i}$ :s are pairwise relatively prime,

$$
\bigcap_{i=1}^{r}\left\langle f_{i}\right\rangle=\left\langle f_{1} \cdots f_{r}\right\rangle,
$$

so Lemma 3.3 shows that $\bigcap \mathcal{D}\left(\left\langle f_{i}\right\rangle\right) \subseteq \mathcal{D}\left(\left\langle f_{1} \cdots f_{r}\right\rangle\right)$.

To show the opposite inclusion, we observe that the polynomials

$$
f=f_{1} \cdots f_{r-1} \quad \text { and } \quad g=f_{r}
$$

are also nonconstant and relatively prime, so the inclusion follows from induction on $r$ if we can prove it for two polynomials. Therefore assume that $f, g \in R$ are nonconstant and relatively prime. We must show that

$$
\mathcal{D}(\langle f g\rangle) \subseteq \mathcal{D}(\langle f\rangle) \cap \mathcal{D}(\langle g\rangle) .
$$

This will be done by induction on the order of $\theta \in \mathcal{D}(\langle f g\rangle)$. If $\theta$ is of order zero, i.e., $\theta \in R$, then $\theta$ preserves any ideal in $R$.

Now assume that the inclusion (7) is true for operators of some order $m \geq 0$, and let $\theta \in \mathcal{D}^{m+1}(\langle f g\rangle)$.

Then $[\theta, g] \in \mathcal{D}^{m}(R)$, and for every $h \in R$ we have

$$
[\theta, g] * h f g=\theta * h f g^{2}-g \theta * h f g \in\langle f g\rangle .
$$

Thus $[\theta, g] \in \mathcal{D}^{m}(\langle f g\rangle)$, and it follows from the induction hypothesis that $[\theta, g] \in$ $\mathcal{D}(\langle f\rangle)$. Thus, for any $h \in R$,

$$
\langle f\rangle \ni[\theta, g] * h f=\theta * h f g-g \theta * h f .
$$

Since $\theta * h f g \in\langle f g\rangle \subseteq\langle f\rangle$, this implies that $g \theta * h f \in\langle f\rangle$. But $f$ and $g$ are relatively prime, so we must have $\theta * h f \in\langle f\rangle$. Repeating the same argument with $f$ and $g$ interchanged shows that we also have $\theta \in \mathcal{D}(\langle g\rangle)$. 


\section{Hyperplane Arrangements}

Definition 4.1. We define a hyperplane, $H$, in $k^{n}$ to be an affine subspace of dimension $(n-1)$. A hyperplane arrangement, $\mathcal{A}$, is a finite set of distinct hyperplanes in $k^{n}$.

Suppose $\mathcal{A}=\bigcup H_{i}$. Each hyperplane $H_{i} \in \mathcal{A}$ is the zeroset of a polynomial $p_{i}$ in $R$ of degree one, defined up to a constant nonzero multiple. We make the convention that whenever we say that polynomials $p_{1}, \ldots, p_{r}$ define a hyperplane arrangement, we mean that they are pairwise relatively prime of degree one, so that $H_{i}$ and $H_{j}$, the hyperplanes defined by $p_{i}$ and $p_{j}$, are distinct if $i \neq j$.

Definition 4.2. The product

$$
P=P(\mathcal{A})=\prod_{H_{i} \in \mathcal{A}} p_{i}
$$

is called a defining polynomial for $\mathcal{A}$. We agree that $P=1$ is the defining polynomial for the empty hyperplane arrangement. The ideal $I=\langle P\rangle$ in $R$ will be called the defining ideal for $\mathcal{A}$.

Unless otherwise explicitly stated, all hyperplane arrangements will be assumed to be nonempty.

Definition 4.3. We call $\mathcal{A}$ centerless if $\bigcap_{H \in \mathcal{A}} H=\emptyset$. If $\bigcap_{H \in \mathcal{A}} H \neq \emptyset$, we call $\mathcal{A}$ centered. If $\mathcal{A}$ is centered, coordinates may be chosen so that each hyperplane contains the origin. In this case we call $\mathcal{A}$ central. We agree that the empty hyperplane arrangement is central.

From now on, we will simply use "arrangement" in place of "hyperplane arrangement". When we do not want to specify if the arrangement under consideration is centerless or not, we will use the term "affine arrangement".

The only (nonempty) central arrangement in $k$ consists of the hyperplane $\{0\}$. Therefore, unless otherwise explicitly stated, we will always assume that $n \geq 2$.

Definition 4.4. Let $\mathcal{A}$ be a central arrangement in $k^{n}$. The intersection lattice, $L(\mathcal{A})$, is the set of all intersections of elements of $\mathcal{A}$. We agree that $k^{n} \in L(\mathcal{A})$, considered as the intersection of the empty family. For $X \in L(\mathcal{A})$ we put $\mathcal{A}_{X}=$ $\{H \in \mathcal{A} \mid X \subseteq H\}$ and we denote by $r(X)$ the codimension of $X$ in $k^{n}$. We say that $\mathcal{A}$ is generic if for every $X \in L(\mathcal{A})$ with $r(X)<n$, we have $\left|\mathcal{A}_{X}\right|=r(X)$, where $\left|\mathcal{A}_{X}\right|$ denotes the cardinality of the set $\mathcal{A}_{X}$.

Example 4.5. For $X \in L(\mathcal{A})$, we have $r(X)=0$ if and only if $X=k^{n}$, and $r(X)=1$ if and only if $X=H \in \mathcal{A}$. Since $\left|\mathcal{A}_{k^{n}}\right|=|\emptyset|=0$ and $\left|\mathcal{A}_{H}\right|=|\{H\}|=$ 1 , it follows that

(i) The empty arrangement is generic for any $n$,

(ii) Any central arrangement in the plane is generic.

(iii) In $k^{3}$, any central arrangement of less than three planes is generic. $A$ central arrangement of three or more planes is generic if and only if any subset of three planes only intersect at the origin.

Since being generic is only defined for central arrangements, we will use "generic arrangement" in place of "generic central arrangement". 


\section{Differential Operators on Hyperplane Arrangements}

We will begin our discussion of differential operators on arrangements with a few results which are valid for affine arrangements. Then, in Section 5.2, we will limit our discussion to central arrangements.

\subsection{Affine Arrangements}

We say that an operator in $\mathcal{D}(R)$ of the form

$$
\sum_{|\alpha|=m} f_{\alpha} \partial^{\alpha}
$$

is of homogeneous order $m$.

From Proposition 2.3 it follows that any $\theta \in \mathcal{D}(R)$ may be uniquely written as a sum,

$$
\theta=\theta_{m}+\ldots+\theta_{0},
$$

of components of homogeneous order, i.e.,

$$
\theta_{i}=\sum_{|\alpha|=i} f_{\alpha} \partial^{\alpha},
$$

for $i=0, \ldots, m$.

Lemma 5.1. Suppose $p \in R$ is a polynomial of degree one, and that $\theta \in \mathcal{D}(R)$. Then

$$
\theta \in \mathcal{D}(\langle p\rangle) \text { if and only if } \theta_{i} \in \mathcal{D}(\langle p\rangle) \text {, for } i=0, \ldots, m \text {, }
$$

where

$$
\theta=\theta_{m}+\ldots+\theta_{0},
$$

is the decomposition of $\theta$ into components of homogeneous order.

Proof. It is trivial that $\theta \in \mathcal{D}(\langle p\rangle)$ if the same thing holds for each $\theta_{i}$. To prove the other implication, we first observe that the homogeneous component $\theta_{0}$ of $\theta$ belongs to $R$, so it preserves any ideal. Thus $\theta_{m}+\ldots+\theta_{1} \in \mathcal{D}(\langle p\rangle)$. Then

$$
\left(\theta_{m}+\ldots+\theta_{1}\right) * p \in\langle p\rangle,
$$

but $\operatorname{deg} p=1$, so

$$
\left(\theta_{m}+\ldots+\theta_{1}\right) * p=\theta_{1} * p \in\langle p\rangle .
$$

By Proposition 3.2, this means that $\theta_{1} \in \mathcal{D}(\langle p\rangle)$. This in turn also means that $\theta_{m}+\ldots+\theta_{2} \in \mathcal{D}(\langle p\rangle)$. In particular, we have

$$
\left(\theta_{m}+\ldots+\theta_{2}\right) * x_{j} p \in\langle p\rangle,
$$

for $j=1, \ldots, n$. Now, $\operatorname{deg} x_{j} p=2$, so

$$
\left(\theta_{m}+\ldots+\theta_{2}\right) * x_{j} p=\theta_{2} * x_{j} p \in\langle p\rangle,
$$

and again Proposition 3.2 shows that $\theta_{2} \in \mathcal{D}(\langle p\rangle)$. Continuing like this we see that each $\theta_{i} \in \mathcal{D}(\langle p\rangle)$, which concludes the proof. 
Notation. If $I$ is an ideal in $R$ we will write $\mathcal{D}^{(m)}(R)$ and $\mathcal{D}^{(m)}(I)$ respectively to denote the operators in $\mathcal{D}(R)$ and $\mathcal{D}(I)$ of homogeneous order $m$. If $A=R / I$, we say that an operator in $\mathcal{D}(A)$ is of homogeneous order $m$ if it is induced by an element in $\mathcal{D}^{(m)}(I)$. Thus, writing $\mathcal{D}^{(m)}(A)$ for the operators in $\mathcal{D}(A)$ of homogeneous order $m$, we have $\mathcal{D}^{(m)}(A) \cong \mathcal{D}^{(m)}(I) / I \mathcal{D}^{(m)}(R)$.

From Lemma 2.2 (i) we know that $\mathcal{D}^{1}(R)=R \oplus \operatorname{Der}(R)$, which implies that

$$
\mathcal{D}^{1}(I)=R \oplus \operatorname{Der}(I)=\mathcal{D}^{(0)}(I) \oplus \mathcal{D}^{(1)}(I),
$$

for any ideal $I$ in $R$. For $m>1$ though, we generally just have a strict inclusion

$$
\bigoplus_{l \leq m} \mathcal{D}^{(l)}(I) \subsetneq \mathcal{D}^{m}(I) .
$$

Example 5.2. Let $I=\left\langle x^{r}\right\rangle \subseteq k[x]$. Then we have, e.g.,

$$
x \partial^{2}-(r-1) \partial \in \mathcal{D}\left(\left\langle x^{r}\right\rangle\right),
$$

but neither $x \partial^{2}$ nor $\partial$ belong to $\mathcal{D}\left(\left\langle x^{r}\right\rangle\right)$ if $r>1$, so

$$
\bigoplus_{l \leq 2} \mathcal{D}^{(l)}(I) \subsetneq \mathcal{D}^{2}(I),
$$

in this case.

This is what makes the following proposition important.

Proposition 5.3. If $I=\left\langle p_{1} \cdots p_{r}\right\rangle$ defines an affine arrangement, then

$$
\mathcal{D}(I)=\bigoplus_{m \geq 0} \mathcal{D}^{(m)}(I),
$$

as an $R$-module.

Proof. We need to show that $\mathcal{D}(I) \subseteq \bigoplus \mathcal{D}^{(m)}(I)$. To do so, assume that $\theta=$ $\theta_{m}+\ldots+\theta_{0} \in \mathcal{D}(I)$, where $\theta_{i}$ is the component of $\theta$ of homogeneous order $i$. We must show that each $\theta_{i}$ also belongs to $\mathcal{D}(I)$.

By Theorem 3.5, $\theta \in \mathcal{D}\left(\left\langle p_{j}\right\rangle\right)$, for $j=1, \ldots, r$. Thus, by Lemma 5.1 , it follows that $\theta_{i} \in \mathcal{D}\left(\left\langle p_{j}\right\rangle\right)$, for each $i=0, \ldots, m$, and $j=1, \ldots, r$. Hence, by Theorem 3.5 again, $\theta_{i} \in \mathcal{D}(I)$, for each $i$.

Corollary 5.4. If $A$ is the coordinate ring of an affine arrangement, then

$$
\mathcal{D}(A)=\bigoplus_{m \geq 0} \mathcal{D}^{(m)}(A),
$$

as an $A$-module.

In view of Proposition 5.3, to find $R$-module generators for $\mathcal{D}(I)$, where $I$ defines an affine arrangement, we may proceed by finding such generators for $\mathcal{D}^{(m)}(I)$, one $m$ at a time.

It will be convenient to study the case of a single hyperplane in some detail, before turning to more general arrangements. 
Lemma 5.5. Let $p \in R$ be a polynomial of degree one. Then $\mathcal{D}^{(m)}(\langle p\rangle)$ is generated, as an $R$-module, by the homogeneous components of highest order of products of $m$ derivations in $\operatorname{Der}(\langle p\rangle)$, together with $\left\{p \partial^{\alpha}|| \alpha \mid=m\right\}$.

Proof. In this case $A=R /\langle p\rangle$ is regular. Therefore, by Lemma 2.2 (iii), $\mathcal{D}(A)$ is generated, as a $k$-algebra, by $A$ and $\operatorname{Der}(A)$, i.e. $\mathcal{D}^{m}(A)$ is generated by $(\operatorname{Der}(A))^{j}, j \leq m$, as an $A$-module.

By Theorem 2.5 the isomorphism $\mathcal{D}(A) \cong \mathcal{D}(\langle p\rangle) /\langle p\rangle \mathcal{D}(R)$ preserves the order filtration on $\mathcal{D}(A)$, so

$$
\operatorname{Der}(A) \cong \operatorname{Der}(\langle p\rangle) /\langle p\rangle \operatorname{Der}(R) .
$$

Hence $\mathcal{D}^{m}(\langle p\rangle)$ is generated by $(\operatorname{Der}(\langle p\rangle))^{j}+\langle p\rangle \mathcal{D}^{m}(R), j \leq m$, as $R$-module. Thus it follows from Proposition 5.3 that $\mathcal{D}^{(m)}(\langle p\rangle)$ is generated by the homogeneous components of highest order of products of $m$ derivations in $\operatorname{Der}(\langle p\rangle)$, together with $\left\{p \partial^{\alpha}|| \alpha \mid=m\right\}$.

Let us now describe the $R$-module $\operatorname{Der}(\langle p\rangle)$. In the following general lemma we do not require that the polynomial $f$ is of degree one.

Lemma 5.6. Let $f$ be any polynomial in $R$ such that there is an element $\delta \in$ $\operatorname{Der}(R)$ with $\delta * f=a f$, for some $a \in k \backslash\{0\}$. Let $\mathcal{N}$ be the $R$-module of derivations annihilating $f$. Then

$$
\operatorname{Der}(\langle f\rangle)=R \delta+\mathcal{N}
$$

Proof. If $\theta \in \operatorname{Der}(R)$, we know from Proposition 3.2 that $\theta \in \operatorname{Der}(\langle f\rangle)$ if and only if $\theta * f \in\langle f\rangle$. Since this holds for $\delta$ it follows immediately that $R \delta+\mathcal{N} \subseteq$ $\operatorname{Der}(\langle f\rangle)$. To show the opposite inclusion, assume $\theta \in \operatorname{Der}(\langle f\rangle)$, say $\theta * f=g f$, $g \in R$. Then

so

$$
\theta * f=g f=\frac{g}{a} \delta * f
$$

$$
\left(\theta-\frac{g}{a} \delta\right) \in \mathcal{N}
$$

This shows that $\theta \in R \delta+\mathcal{N}$.

Suppose $\delta=\sum g_{i} \partial_{i}$ is such that $\delta * f=a f$, as in the lemma. Then

$$
f=\frac{1}{a} \delta * f=\frac{1}{a} \sum g_{i} \partial_{i} * f \in\left\langle\partial_{1} * f, \ldots, \partial_{n} * f\right\rangle .
$$

Conversely, if $f \in\left\langle\partial_{1} * f, \ldots, \partial_{n} * f\right\rangle$, say $f=\sum g_{i} \partial_{i} * f$, then

$$
a f=a \sum g_{i} \partial_{i} * f=\delta * f,
$$

with $\delta=a \sum g_{i} \partial_{i}$. Hence a derivation as in the lemma exists if and only if $f \in\left\langle\partial_{1} * f, \ldots, \partial_{n} * f\right\rangle$. This is obviously the case if $\left\langle\partial_{1} * f, \ldots, \partial_{n} * f\right\rangle=R$, which holds for any $f$ of degree one, i.e., for any polynomial defining a hyperplane.

Recall that a polynomial $f \in R$ is called quasihomogeneous if there are integers $t_{1}, \ldots, t_{n} \geq 0$, not all zero, such that $f$ is homogeneous with respect to $\operatorname{deg} x^{\alpha}=t_{1} \alpha_{1}+\ldots+t_{n} \alpha_{n}$. We have $f \in\left\langle\partial_{1} * f, \ldots, \partial_{n} * f\right\rangle$ for any polynomial $f$ which, possibly after a translation, is quasihomogeneous.

When $p$ is a polynomial of degree one, the module $\mathcal{N}$ in Lemma 5.6 is particularly nice. 
Definition 5.7. Let $V$ be the $k$-vector space

$$
V=\sum_{i=1}^{n} k \partial_{i}
$$

and, if $H$ is a hyperplane through the origin in $k^{n}$, define $V_{H} \subseteq V$ to be the $(n-1)$-dimensional subspace

$$
V_{H}=\left\{\sum_{i=1}^{n} b_{i} \partial_{i} \mid\left(b_{1}, \ldots, b_{n}\right) \in H\right\} .
$$

If $p$ is any polynomial of degree one, then $p-p(0)$ is a nonzero linear form, which is equal to $p$ in case $p$ is already homogeneous, so $p-p(0)$ defines a hyperplane through the origin.

Lemma 5.8. Let $p$ be a polynomial of degree one, and let $\mathcal{N}$ be the module of derivations annihilating $p$. Let $H$ be the hyperplane through the origin defined by $p-p(0)$. Then

$$
\mathcal{N}=R V_{H}
$$

Proof. Suppose $p=a_{0}+\sum a_{i} x_{i}$. A vector $\left(b_{1}, \ldots, b_{n}\right) \in k^{n}$ lies in $H$ if and only if $\sum a_{i} b_{i}=0$, i.e., if and only if $\left(b_{1}, \ldots, b_{n}\right)$ is a syzygy of $\left(a_{1}, \ldots, a_{n}\right)$. In fact, the vectors in $H$ generate the whole $R$-module of syzygies of $\left(a_{1}, \ldots, a_{n}\right)$.

On the other hand, a derivation $\delta=\sum f_{i} \partial_{i} \in \operatorname{Der}(R)$ lies in $\mathcal{N}$ if and only if $0=\delta * p=\sum f_{i} a_{i}$, i.e., if and only if $\left(f_{1}, \ldots, f_{n}\right)$ is a syzygy of $\left(a_{1}, \ldots, a_{n}\right)$. Hence $\mathcal{N}=R V_{H}$.

Thus, let $p$ be a polynomial of degree one, and $H$ the hyperplane defined by $p-p(0)$. If we choose a basis $\left\{\delta_{1}, \ldots, \delta_{n-1}\right\}$ for the vector space $V_{H}$ in Definition 5.7, we may extend this to a basis $\left\{\delta_{1}, \ldots, \delta_{n}\right\}$ for $V$.

Since $\delta_{n}$ does not lie in the span of $\delta_{1}, \ldots, \delta_{n-1}$ it cannot annihilate $p$. Whence $p \delta_{n} \in \operatorname{Der}(R)$ meets the requirements of Lemma 5.6, and it follows that $\operatorname{Der}(\langle p\rangle)$ is generated by $\left\{\delta_{1}, \ldots, \delta_{n-1}, p \delta_{n}\right\}$.

We may now improve on Lemma 5.5:

Proposition 5.9. Suppose $p \in R$ is a polynomial of degree one. Let $H$ be the hyperplane through the origin defined by the polynomial $p-p(0)$, and let $M=\left\{\delta_{1}, \ldots, \delta_{n}\right\}$ be a basis for $V$ such that $\left\{\delta_{1}, \ldots, \delta_{n-1}\right\}$ is a basis for the subspace $V_{H}$ from Definition 5.7. Then

$$
\mathcal{D}^{(m)}(\langle p\rangle)=\sum_{\substack{|\alpha|=m \\ \alpha_{n}=0}} R \delta^{\alpha}+\sum_{\substack{|\alpha|=m \\ \alpha_{n}>0}} R p \delta^{\alpha},
$$

as an $R$-module.

Proof. We know that $\operatorname{Der}(\langle p\rangle)$ is generated by $\left\{\delta_{1}, \ldots, \delta_{n-1}, p \delta_{n}\right\}$. Thus, by Lemma $5.5, \mathcal{D}^{(m)}(\langle p\rangle)$ is generated by the homogeneous components of highest order of products of $m$ of these derivations, together with $\left\{p \partial^{\beta}|| \beta \mid=m\right\}$.

The homogeneous component of highest order of $\left(p \delta_{n}\right)^{l}$ is $p^{l} \delta_{n}^{l}$. Since the derivations $\delta_{1}, \ldots, \delta_{n}$ belong to $V$ they all commute with each other and since 
$\delta_{i} * p=0$ for $i=1, \ldots, n-1$, it follows that $\delta_{1}, \ldots, \delta_{n-1}$ commute with $p \delta_{n}$. Thus the homogeneous component of highest order of a product of the derivations $\delta_{1}, \ldots, \delta_{n-1}, p \delta_{n}$ is of the form $p^{\alpha_{n}} \delta^{\alpha}$. Any such element is clearly a multiple of one of the proposed generators.

Since $\left\{\delta_{1}, \ldots, \delta_{n}\right\}$ is a basis for $V$ it follows that any $\partial^{\beta}$ with $|\beta|=m$ may be expressed as a $k$-linear combination of $\left\{\delta^{\alpha}|| \alpha \mid=m\right\}$, so any element of the form $p \partial^{\beta},|\beta|=m$, is an $R$-linear combination of the proposed generators.

We end this section with a few examples where we use the tools we have developed so far to find the differential operators on some affine arrangements.

Example 5.10. Suppose that $I=\left\langle p_{1} \cdots p_{r}\right\rangle$ defines an affine arrangement of parallel hyperplanes. Then there is a nonzero linear form $p$ such that

$$
p_{i}=p+a_{i}, i=1, \ldots, r
$$

where $a_{i} \in k$, and $a_{i} \neq a_{j}$ if $i \neq j$.

Let $H$ be the hyperplane through the origin defined by $p$, and let $\mathcal{N}_{i}$ be the $R$-module of derivations annihilating $p_{i}$, for $i=1, \ldots, r$. Then, by Lemma 5.8,

$$
\mathcal{N}_{1}=\ldots=\mathcal{N}_{r}=R V_{H}
$$

so letting $M=\left\{\delta_{1}, \ldots, \delta_{n}\right\}$ be a basis for $V$ such that $\left\{\delta_{1}, \ldots, \delta_{n-1}\right\}$ is a basis for $V_{H}$, it follows from Proposition 5.9 that

$$
\mathcal{D}^{(m)}\left(\left\langle p_{i}\right\rangle\right)=\sum_{\substack{|\alpha|=m \\ \alpha_{n}=0}} R \delta^{\alpha}+\sum_{\substack{|\alpha|=m \\ \alpha_{n}>0}} R p_{i} \delta^{\alpha},
$$

for $i=1, \ldots, r$.

The set $\left\{\delta^{\alpha}|| \alpha \mid=m\right\}$ is a basis, and the $p_{i}$ :s are pairwise relatively prime. Thus, by Theorem 3.5,

$$
\mathcal{D}^{(m)}(I)=\bigcap_{i=1}^{r} \mathcal{D}^{(m)}\left(\left\langle p_{i}\right\rangle\right)=\sum_{\substack{|\alpha|=m \\ \alpha_{n}=0}} R \delta^{\alpha}+\sum_{\substack{|\alpha|=m \\ \alpha_{n}>0}} I \delta^{\alpha} .
$$

Let $A=R / I$. Then Proposition 5.3 and Theorem 2.5 give that

$$
\mathcal{D}(A)=A\left[\delta_{1}, \ldots, \delta_{n-1}\right],
$$

where the $\delta_{i}: s$ commute with each other, but not with elements in the coefficient ring $A$. In this case, $\mathcal{D}(A)$ is an Ore extension of $A$.

Remark. After a linear change of coordinates we could have assumed that $p=$ $x_{1}$, so that $P=p_{1} \cdots p_{r}$ would be a polynomial of degree $r$ in one variable, with $r$ distinct zeros in $k$. Therefore we could have reached the result in Example 5.10 by other, well known, methods. However, this example illustrates how our results, in particular Theorem 3.5 and Proposition 5.3, may be used.

Example 5.11. Suppose that $\mathcal{A}$ is an affine arrangement of three lines in the plane, defined by $I=\left\langle p_{1} p_{2} p_{3}\right\rangle \subseteq k[x, y]=R$, and let $A=R / I$. There are four possibilities: 
(i) Three parallel lines.

This case follows from Example 5.10.

(ii) Two parallel lines intersecting a third.

Suppose that the lines defined by $p_{1}$ and $p_{2}$ are parallel to each other, but not to the line defined by $p_{3}$.

Let $M=\left\{\delta_{1}, \delta_{2}\right\}$ be a basis for $V=k \partial_{x}+k \partial_{y}$ such that

$$
\delta_{1} * p_{1}=\delta_{1} * p_{2}=\delta_{2} * p_{3}=0,
$$

but $\delta_{1} * p_{3}, \delta_{2} * p_{1}$, and $\delta_{2} * p_{2}$ are all nonzero. Then

$$
\mathcal{D}^{(m)}\left(\left\langle p_{1} p_{2}\right\rangle\right)=R \delta_{1}^{m}+\sum_{j=0}^{m-1} R p_{1} p_{2} \delta_{1}^{j} \delta_{2}^{m-j},
$$

by Example 5.10, and

$$
\mathcal{D}^{(m)}\left(\left\langle p_{3}\right\rangle\right)=\sum_{j=1}^{m} R p_{3} \delta_{1}^{j} \delta_{2}^{m-j}+R \delta_{2}^{m},
$$

by Proposition 5.9. As in Example 5.10, the set $\left\{\delta_{1}^{j} \delta_{2}^{m-j} \mid j=0, \ldots, m\right\}$ is a basis, and $p_{1} p_{2}$ is relatively prime to $p_{3}$, so from Theorem 3.5 it follows that

$$
\begin{aligned}
\mathcal{D}^{(m)}(I) & =\mathcal{D}^{(m)}\left(\left\langle p_{1} p_{2}\right\rangle\right) \cap \mathcal{D}^{(m)}\left(\left\langle p_{3}\right\rangle\right) \\
& =R p_{3} \delta_{1}^{m}+R p_{1} p_{2} \delta_{2}^{m}+\sum_{j=1}^{m-1} I \delta_{1}^{j} \delta_{2}^{m-j}
\end{aligned}
$$

for $m \geq 1$. Hence, again by Proposition 5.3 and Theorem 2.5,

$$
\mathcal{D}(A)=A+\bigoplus_{m \geq 1}\left(A \overline{p_{3}} \delta_{1}^{m}+A \overline{p_{1} p_{2}} \delta_{2}^{m}\right) .
$$

(iii) Three nonparallel lines with no common point of intersection.

After a translation we may assume that two of the lines intersect at the origin. Then, after a linear change of coordinates, we may assume that the three lines are defined by the ideal $I=\langle x y(x-y+1)\rangle$.

With $M=\left\{\partial_{x}, \partial_{y}\right\}$ it follows from Proposition 5.9, that

$$
\mathcal{D}^{(m)}(\langle x\rangle)=\sum_{j=1}^{m} R x \partial_{x}^{j} \partial_{y}^{m-j}+R \partial_{y}^{m},
$$

and

$$
\mathcal{D}^{(m)}(\langle y\rangle)=R \partial_{x}^{m}+\sum_{j=0}^{m-1} R y \partial_{x}^{j} \partial_{y}^{m-j}
$$


so, by Theorem 3.5, we have

$$
\mathcal{D}^{(m)}(\langle x y\rangle)=R x \partial_{x}^{m}+\sum_{j=1}^{m-1} R x y \partial_{x}^{j} \partial_{y}^{m-j}+R y \partial_{y}^{m} .
$$

From Proposition 5.9 it follows, with $M=\left\{\partial_{x}+\partial_{y}, \partial_{x}\right\}$, that

$$
\begin{aligned}
\mathcal{D}^{(m)}(\langle x-y+1\rangle) & =R\left(\partial_{x}+\partial_{y}\right)^{m}+\sum_{j=1}^{m} R(x-y+1) \partial_{x}^{j}\left(\partial_{x}+\partial_{y}\right)^{m-j} \\
& =R\left(\partial_{x}+\partial_{y}\right)^{m}+\sum_{j=1}^{m} R(x-y+1) \partial_{x}^{j} \partial_{y}^{m-j} .
\end{aligned}
$$

Applying Theorem 3.5 again gives

$$
\begin{aligned}
\mathcal{D}^{(m)}(I)= & \mathcal{D}^{(m)}(\langle x y\rangle) \cap \mathcal{D}^{(m)}(\langle x-y+1\rangle) \\
= & R x(x-y+1) \partial_{x}^{m}+R x y\left(\partial_{x}+\partial_{y}\right)^{m}+R y(x-y+1) \partial_{y}^{m} \\
& +\sum_{j=1}^{m-1} I \partial_{x}^{j} \partial_{y}^{m-j}
\end{aligned}
$$

for $m \geq 1$. Thus

$$
\begin{aligned}
\mathcal{D}(A)= & A \\
& +\bigoplus_{m \geq 1}\left(\overline{A \overline{x(x-y+1)}} \partial_{x}^{m}+A \overline{x y}\left(\partial_{x}+\partial_{y}\right)^{m}+A \overline{y(x-y+1)} \partial_{y}^{m}\right) .
\end{aligned}
$$

(iv) Three lines intersecting in one point.

After a translation we may assume that the common point of intersection is the origin. This case will follow from Corollary 5.31 in Section 5.2.1.

\subsection{Central Arrangements}

Let us now concentrate on central arrangements. In this case, if $\mathcal{A}$ is defined by $p_{1}, \ldots, p_{r}$, each $p_{i}$ is a nonzero linear form, and the defining polynomial $P(\mathcal{A})$ is a homogeneous polynomial whose degree is the cardinality of $\mathcal{A}$.

There is a derivation preserving any homogeneous ideal, namely the Euler derivation,

$$
\varepsilon=\sum_{i=1}^{n} x_{i} \partial_{i}
$$

This derivation is easily seen to have the property

$$
\varepsilon * f=r f
$$

if $f$ is homogeneous of degree $r$. In particular, $\varepsilon$ preserves any ideal defining a central arrangement. By Lemma 5.5, we need to know what the component of highest order of a power of the Euler derivation looks like. 
Let $h=\sum_{i=1}^{n} x_{i} y_{i} \in k\left[x_{1}, \ldots, x_{n}, y_{1}, \ldots, y_{n}\right]$. Mapping $y_{i}$ to $\partial_{i}$ gives $\varepsilon$. For $m \in \mathbb{N}$ we have $h^{m}=\sum_{|\alpha|=m} \frac{m !}{\alpha !} x^{\alpha} y^{\alpha}$, and again mapping $y_{i}$ to $\partial_{i}$ gives an operator

$$
\varepsilon_{m}=\sum_{|\alpha|=m} \frac{m !}{\alpha !} x^{\alpha} \partial^{\alpha} .
$$

We see that $\varepsilon_{0}=1$ and $\varepsilon_{1}=\varepsilon$, but for $m \neq 0,1$ we have of course that $\varepsilon_{m} \neq \varepsilon^{m}$. However

\section{Lemma 5.12.}

(i) $\varepsilon_{m+1}=\varepsilon_{m}(\varepsilon-m)$.

(ii) $\varepsilon_{m}=\varepsilon(\varepsilon-1) \cdots(\varepsilon-m+1)$, if $m>0$.

(iii) $\varepsilon_{m}$ is the homogeneous component of highest order of $\varepsilon^{m}$.

(iv) If $f \in R$ is homogeneous of degree $r$, then

$$
\varepsilon_{m} * f= \begin{cases}\frac{r !}{(r-m) !} f & \text { if } m \leq r, \\ 0 & \text { if } m>r .\end{cases}
$$

Proof. It is easily seen that $(i) \Rightarrow(i i) \Rightarrow(i i i)$. Condition (iv) is obvious for $m=0$, and for $m>0$ this too follows from $(i i)$, because

$$
\varepsilon_{m} * f=\varepsilon(\varepsilon-1) \cdots(\varepsilon-m+1) * f=r(r-1) \cdots(r-m+1) f,
$$

since $\varepsilon * f=r f$.

It remains to show $(i)$. This is clear if $m=0$. If $m>0$ we get

$$
\begin{aligned}
\varepsilon_{m} \cdot \varepsilon & =\sum_{|\alpha|=m} \sum_{i=1}^{n} \frac{m !}{\alpha !} x^{\alpha} \partial^{\alpha} x_{i} \partial_{i}=\sum_{|\alpha|=m} \sum_{i=1}^{n} \frac{m !}{\alpha !} x^{\alpha}\left(x_{i} \partial^{\alpha}+\alpha_{i} \partial^{\alpha-e_{i}}\right) \partial_{i} \\
& =\sum_{|\alpha|=m} \sum_{i=1}^{n} \frac{m !}{\alpha !} x^{\alpha+e_{i}} \partial^{\alpha+e_{i}}+\sum_{|\alpha|=m} \sum_{i=1}^{n} \frac{m !}{\alpha !} \alpha_{i} x^{\alpha} \partial^{\alpha} \\
& =\sum_{|\beta|=m+1} \sum_{i=1}^{n} \beta_{i} \frac{m !}{\beta !} x^{\beta} \partial^{\beta}+\sum_{|\alpha|=m} \frac{m !}{\alpha !}\left(\sum_{i=1}^{n} \alpha_{i}\right) x^{\alpha} \partial^{\alpha} \\
& =\sum_{|\beta|=m+1}(m+1) \frac{m !}{\beta !} x^{\beta} \partial^{\beta}+m \sum_{|\alpha|=m} \frac{m !}{\alpha !} x^{\alpha} \partial^{\alpha} \\
& =\sum_{|\beta|=m+1} \frac{(m+1) !}{\beta !} x^{\beta} \partial^{\beta}+m \varepsilon_{m}=\varepsilon_{m+1}+m \varepsilon_{m} .
\end{aligned}
$$

\subsubsection{Module Generators}

In this section we will find generators for $\mathcal{D}(I)$ as a module over $R$, when $I$ defines a generic arrangement. By Theorem 2.5, this will give $A$-module generators for $\mathcal{D}(A)$, if $A=R / I$ is the coordinate ring of the arrangement. 
First suppose that $r \geq 1$ and that $p_{1}, \ldots, p_{r}$ define a central arrangement. Let $H_{i}$ be the hyperplane defined by $p_{i}$, for $i=1, \ldots, r$, and let $V_{i}=V_{H_{i}} \subseteq V$ be the subspace generating the module of derivations annihilating $p_{i}$.

If $\left\{i_{1}, \ldots, i_{s}\right\} \subseteq\{1, \ldots, r\}$, then an element $\delta \in V_{i_{1}} \cap \ldots \cap V_{i_{s}}$ annihilates $p_{i_{1}} \cdots p_{i_{s}}$. Thus, if $\left\{i_{s+1}, \ldots, i_{r}\right\}=\{1, \ldots, r\} \backslash\left\{i_{1}, \ldots, i_{s}\right\}$, then the derivation $p_{i_{s+1}} \cdots p_{i_{r}} \delta$ preserves the ideal $\left\langle p_{1} \cdots p_{r}\right\rangle$. We will show that if the arrangement defined by $\left\langle p_{1} \cdots p_{r}\right\rangle$ is generic, then $\mathcal{D}^{(m)}\left(\left\langle p_{1} \cdots p_{r}\right\rangle\right)$ is generated by $\varepsilon_{m}$ together with elements of the form $p_{i_{s+1}} \cdots p_{i_{r}} \theta$, where $\theta$ is a product of $m$ derivations in $V_{i_{1}} \cap \ldots \cap V_{i_{s}}$.

To do so we should have a basis for each intersection of $V_{i}:$ s, including the whole of $V$, considered as the intersection of the empty family. Therefore, assume that $p_{1}, \ldots, p_{r}$ define a generic arrangement. Since each $V_{i}$ is isomorphic to $H_{i}$, and since $\mathcal{A}=\bigcup H_{i}$ is generic, we know that

$$
\operatorname{dim}\left(V_{i_{1}} \cap \ldots \cap V_{i_{s}}\right)=\operatorname{dim}\left(H_{i_{1}} \cap \ldots \cap H_{i_{s}}\right)= \begin{cases}n-s & \text { if } s<n, \\ 0 & \text { if } s \geq n .\end{cases}
$$

We will now show how to find a subset $M$ of $V$ containing a basis for each intersection of $V_{i}$ :s.

Lemma 5.13. Let $p_{1}, \ldots, p_{r}$ be linear forms defining a generic arrangement.

Choose a subset $M$ of $V$ as follows.

If $r<n$ : choose a basis $\left\{\delta_{r+1}, \ldots, \delta_{n}\right\}$ for $\bigcap_{i=1}^{r} V_{i}$. Then, for each $i=1, \ldots, r$, choose an element $\delta_{i}$ so that $\left\{\delta_{i}, \delta_{r+1}, \ldots, \delta_{n}\right\}$ is a basis for $\bigcap_{j \neq i} V_{j}$, and let $M=\left\{\delta_{1}, \ldots, \delta_{n}\right\}$.

If $r \geq n$ : choose a basis element for each intersection of $n-1$ of the $V_{i}: s$, and let $M=\left\{\delta_{1}, \ldots, \delta_{t}\right\}$ be the set of all of these.

$A$ set $M$ chosen in this way contains a basis for each intersection of $V_{i}: s$, including the whole of $V$.

Proof. The case $r>n$ follows from the case $r=n$. Any intersection $V_{i_{1}} \cap \ldots \cap V_{i_{s}}$ with $s \geq n$ is zero, and for an intersection $V_{i_{1}} \cap \ldots \cap V_{i_{s}}$ with $s \leq n-1$, choose any $V_{i_{s+1}}, \ldots, V_{i_{n}}$ to give $n V_{i}$ :s. The subset of $n$ elements of $M$ which are bases for the intersections of $n-1$ of these $V_{i}$ :s contains a basis for $V_{i_{1}} \cap \ldots \cap V_{i_{s}}$ by the case $r=n$.

Now suppose that $r \leq n$. In this case $M=\left\{\delta_{1}, \ldots, \delta_{n}\right\}$, and an intersection

$$
V_{i_{1}} \cap \ldots \cap V_{i_{s}}
$$

with $1 \leq s \leq r$, has dimension $n-s$ and contains $\delta_{i_{s+1}}, \ldots, \delta_{i_{r}}, \delta_{r+1}, \ldots, \delta_{n}$, so these form a basis if they are linearly independent. Hence we are done if we can show that $M$ is a basis for $V$, i.e., that $\delta_{1}, \ldots, \delta_{n}$ are linearly independent.

To do so, assume $\sum a_{i} \delta_{i}=0$. We have numbered the $\delta_{i}$ :s so that

$$
\delta_{i} * p_{j}= \begin{cases}b_{j} \in k \backslash\{0\} & \text { if } i=j, \\ 0 & \text { if } i \neq j .\end{cases}
$$

Thus

$$
0=\left(\sum a_{i} \delta_{i}\right) * p_{j}=a_{j} b_{j}
$$


for each $j=1, \ldots, r$, so $a_{i}=0$ for $i=1, \ldots, r$. This finishes the proof for the case $r=n$. If $r<n$ this shows that $\sum_{i=r+1}^{n} a_{i} \delta_{i}=0$, but $\left\{\delta_{r+1}, \ldots, \delta_{n}\right\}$ is a basis, so we must have $a_{i}=0$ for $i=r+1, \ldots, n$, too.

Remark. When $r=1$, this gives a set $M$ as in Proposition 5.9, although with a different numbering of the basis elements. As in Proposition 5.9, the set $M$ in Lemma 5.13 is not unique. However, we will use $M$ to define certain $R$ submodules of $\mathcal{D}(R)$, in Definition 5.15, and as we shall see in Lemma 5.17, given $p_{1}, \ldots, p_{r}$, any choice of basis elements will give the same module, as long as they are chosen as in the lemma. Therefore we will feel justified to refer to the set $M$ from Lemma 5.13 .

To find bases for intersections of $V_{i}: \mathrm{s}$ is easy. Because of the isomorphism between $V_{H}$ and $H$, finding a basis for $V_{i_{1}} \cap \ldots \cap V_{i_{s}}$ amounts to the same thing as finding the solutions of the system

$$
p_{i_{1}}=\ldots=p_{i_{s}}=0 .
$$

Let us now define a new subset of $\operatorname{Der}(R)$, associated to a generic arrangement. Actually, this is a subset of $\operatorname{Der}(I)$, as we shall see in Proposition 5.18.

Definition 5.14. Suppose $p_{1}, \ldots, p_{r}$ are linear forms defining a generic arrangement. We define a subset $D$ of $\operatorname{Der}(R)$ as

$$
D=\left\{P_{1} \delta_{1}, \ldots, P_{s} \delta_{s}\right\},
$$

where $\left\{\delta_{1}, \ldots, \delta_{s}\right\}$ is the set $M$ from Lemma 5.13, and where each $P_{i}$ is the product of those $p_{j}: s$ which are not annihilated by $\delta_{i}$. If all $p_{j}: s$ are annihilated by $\delta_{i}$, we put $P_{i}=1$.

Remark. The set $D$ depends on the set $M$ from Lemma 5.13, so it is not uniquely defined. However, to choose some other basis element $\gamma_{i}$ instead of $\delta_{i}$ in $M$, will not change the coefficient $P_{i}$, i.e., we will get $P_{i} \gamma_{i}$ instead of $P_{i} \delta_{i}$ in $D$, since $\delta_{i}$ and $\gamma_{i}$ annihilate the same $p_{j}$ :s. Just as for the set $M$, we will refer to the set $D$ from Definition 5.14.

For any ideals $I, J$ in $R$, we define their ideal quotient as

$$
(I: J)=\{f \in R \mid f J \subseteq I\} .
$$

If $J=\langle f\rangle$ we will simply write $(I: f)$ for $(I: J)$.

We may now make the following definition.

Definition 5.15. Suppose $I=\left\langle p_{1} \cdots p_{r}\right\rangle$ defines a generic arrangement, and that

$$
D=\left\{P_{1} \delta_{1}, \ldots, P_{s} \delta_{s}\right\}
$$

is the set associated to $I$ according to Definition 5.14. Then we define an $R$ module $\Theta^{m}(I)$ by

$$
\Theta^{m}(I)=\sum_{|\alpha|=m}\left(I:\left(I: P^{\alpha}\right)\right) \delta^{\alpha}+R \varepsilon_{m},
$$

for each $m \in \mathbb{N}$. 
Observe that, in the definition above, the multi-indices $\alpha$ belong to $\mathbb{N}^{s}$, where $s>n$ if $r>n$.

We also observe that $\left(I:\left(I: P^{\alpha}\right)\right)$ is a principal ideal in $R$. More precisely, if $P^{\alpha}=1$, then $\left(I:\left(I: P^{\alpha}\right)\right)=R$, and if

$$
P^{\alpha}=p_{i_{1}}^{a_{1}} \cdots p_{i_{t}}^{a_{t}}
$$

with all $a_{j} \neq 0$, then

$$
\left(I:\left(I: P^{\alpha}\right)\right)=\left\langle p_{i_{1}} \cdots p_{i_{t}}\right\rangle .
$$

This follows from unique factorization, since the $p_{i}$ :s are pairwise relatively prime. The generator $p_{i_{1}} \cdots p_{i_{t}}$ of $\left(I:\left(I: P^{\alpha}\right)\right)$ is the product of those $p_{j}: \mathrm{s}$ such that some $\delta_{i}$ with $\alpha_{i} \neq 0$ does not annihilate $p_{j}$.

For ease of notation, let us make the following

Definition 5.16. Suppose $I=\left\langle p_{1} \cdots p_{r}\right\rangle$ defines a generic arrangement and that $\left\{\delta_{1}, \ldots, \delta_{s}\right\}$ is the set $M$ associated to I according to Lemma 5.13.

For each $\alpha \in \mathbb{N}^{s}$, define $P_{\alpha}$ as the product of those $p_{j}$ :s such that some $\delta_{i}$ with $\alpha_{i} \neq 0$ does not annihilate $p_{j}$. If every $\delta_{i}$ with $\alpha_{i} \neq 0$ annihilate all $p_{j}: s$, we define $P_{\alpha}=1$.

Thus, for each $\alpha \in \mathbb{N}^{s}$, we have

$$
R P_{\alpha}=\left(I:\left(I: P^{\alpha}\right)\right),
$$

so

$$
\Theta^{m}(I)=\sum_{|\alpha|=m} R P_{\alpha} \delta^{\alpha}+R \varepsilon_{m} .
$$

Finally we observe that, although the set $\left\{P_{\alpha} \delta^{\alpha}|| \alpha \mid=m\right\} \cup\left\{\varepsilon_{m}\right\}$ of generators for $\Theta^{m}(I)$ is finite, it is in general not minimal. A precise statement for the case $m=1$ may be found in [7].

All results from Lemma 5.17 to Lemma 5.27, inclusive, are obvious for $m=0$, so in the proofs of all of these results, we may without loss of generality assume that $m \geq 1$.

Lemma 5.17. If $I=\left\langle p_{1} \cdots p_{r}\right\rangle$ defines a generic arrangement, then different choices of basis elements in $M$ in Lemma 5.13 give the same $R$-module $\Theta^{m}(I)$, for each $m$.

Proof. This is obvious for $r \geq n$, since the elements in $M$ are bases for onedimensional spaces in this case, so that they are unique up to nonzero constants. Therefore we may assume that $r<n$. If

$$
M_{\delta}=\left\{\delta_{1}, \ldots, \delta_{n}\right\} \text { and } M_{\gamma}=\left\{\gamma_{1}, \ldots, \gamma_{n}\right\}
$$

are chosen as in Lemma 5.13, the sets $D$ from Definition 5.14 will be

$$
D_{\delta}=\left\{P_{1} \delta_{1}, \ldots, P_{n} \delta_{n}\right\}=\left\{p_{1} \delta_{1}, \ldots, p_{r} \delta_{r}, \delta_{r+1}, \ldots, \delta_{n}\right\}
$$


and

$$
D_{\gamma}=\left\{P_{1} \gamma_{1}, \ldots, P_{n} \gamma_{n}\right\}=\left\{p_{1} \gamma_{1}, \ldots, p_{r} \gamma_{r}, \gamma_{r+1}, \ldots, \gamma_{n}\right\} .
$$

Let $\Theta_{\delta}^{m}(I)$ and $\Theta_{\gamma}^{m}(I)$ be the modules given by $D_{\delta}$ and $D_{\gamma}$ according to Definition 5.15. By symmetry, to see that $\Theta_{\delta}^{m}(I)=\Theta_{\gamma}^{m}(I)$, it is enough to show that $\Theta_{\delta}^{m}(I) \subseteq \Theta_{\gamma}^{m}(I)$.

Since

$$
\left\{\delta_{r+1}, \ldots, \delta_{n}\right\} \text { and }\left\{\gamma_{r+1}, \ldots, \gamma_{n}\right\}
$$

and, for $i=1, \ldots, r$,

$$
\left\{\delta_{i}, \delta_{r+1}, \ldots, \delta_{n}\right\} \text { and }\left\{\gamma_{i}, \gamma_{r+1}, \ldots, \gamma_{n}\right\},
$$

respectively, are bases for the same vectorspaces, there are $a_{i}, a_{i j} \in k$ such that

$$
\delta_{i}=a_{i} \gamma_{i}+\sum_{j=r+1}^{n} a_{i j} \gamma_{j}
$$

for $i=1, \ldots, r$, and

$$
\delta_{i}=\sum_{j=r+1}^{n} a_{i j} \gamma_{j},
$$

for $i=r+1, \ldots, n$. Thus, if $|\alpha|=m$,

$$
\delta^{\alpha}=a \gamma^{\alpha}+\sum_{|\beta|=m} a_{\beta} \gamma^{\beta},
$$

where $a, a_{\beta} \in k$, and $a_{\beta} \neq 0$ only if $\gamma^{\beta}$ is a product of $\gamma_{r+1}, \ldots, \gamma_{n}$, and the $\gamma_{i}$ :s with $\alpha_{i} \neq 0$, i.e., such that $P_{\alpha}$ is a multiple, $f_{\beta} P_{\beta}$, of $P_{\beta}$, where $f_{\beta}$ is 1 , or a product of $p_{j}$ :s. Hence

$$
\begin{aligned}
P_{\alpha} \delta^{\alpha} & =a P_{\alpha} \gamma^{\alpha}+\sum_{|\beta|=m} a_{\beta} P_{\alpha} \gamma^{\beta} \\
& =a P_{\alpha} \gamma^{\alpha}+\sum_{|\beta|=m} a_{\beta} f_{\beta} P_{\beta} \gamma^{\beta} \in \Theta_{\gamma}^{m}(I),
\end{aligned}
$$

for all $\alpha$ with $|\alpha|=m$.

We will show that $\mathcal{D}^{(m)}(I)=\Theta^{m}(I)$. To begin with we have

Proposition 5.18. Suppose that $I=\left\langle p_{1} \cdots p_{r}\right\rangle$ defines a generic arrangement. Then

$$
\Theta^{m}(I) \subseteq \mathcal{D}^{(m)}(I),
$$

as $R$-modules. 
Proof. From Lemma 5.12, (iv), it follows that $R \varepsilon_{m} \subseteq \mathcal{D}^{(m)}(I)$. To see that

$$
\sum_{|\alpha|=m} R P_{\alpha} \delta^{\alpha} \subseteq \mathcal{D}^{(m)}(I),
$$

we observe that, if $f \in R$ and $\theta$ is a derivation annihilating $f$, we have $\theta^{l} * g f=$ $\left(\theta^{l} * g\right) f$, for any $g \in R$. Thus $\delta^{\alpha}$ preserves the ideal generated by the product of those $p_{j}$ :s that all $\delta_{i}$ with $\alpha_{i} \neq 0$ annihilate. It follows that $P_{\alpha} \delta^{\alpha}$ preserves $\left\langle p_{1} \cdots p_{r}\right\rangle$, i.e., (8) holds.

To show that $\mathcal{D}^{(m)}(I) \subseteq \Theta^{m}(I)$, we will use a method for doing induction on the cardinality of an arrangement $\mathcal{A}$, known as deletion and restriction. Let us therefore make the following definition.

Definition 5.19. Let $\mathcal{A}$ be a central arrangement and let $H \in \mathcal{A}$ be a fixed hyperplane. Let $\mathcal{A}^{\prime}=\mathcal{A} \backslash\{H\}$ and let $\mathcal{A}^{\prime \prime}=\left\{K \cap H \mid K \in \mathcal{A}^{\prime}\right\}$. Then $\mathcal{A}^{\prime}$ is called a deleted arrangement and $\mathcal{A}^{\prime \prime}$ is called a restricted arrangement. We call $H$ the distinguished hyperplane.

Observe that if $\mathcal{A}$ is a generic arrangement, so are $\mathcal{A}^{\prime}$ and $\mathcal{A}^{\prime \prime}$. For a fixed hyperplane $H \in \mathcal{A}$, we may assume, after a linear change of coordinates, that $H$ is defined by the equation $x_{1}=0$.

Therefore, suppose $\mathcal{A}$ is defined by the ideal $I=\left\langle x_{1} p_{1} \cdots p_{r}\right\rangle$, and let the distinguished hyperplane be the hyperplane defined by $x_{1}$. Let $I^{\prime}=\left\langle p_{1} \cdots p_{r}\right\rangle$, and let $I^{\prime \prime}=\left\langle q_{1} \cdots q_{r}\right\rangle \subseteq k\left[x_{2}, \ldots, x_{n}\right]$, where

$$
q_{i}\left(x_{2}, \ldots, x_{n}\right)=p_{i}\left(0, x_{2}, \ldots, x_{n}\right), i=1, \ldots, r .
$$

Then $I^{\prime}$ is the defining ideal for $\mathcal{A}^{\prime}$ and $\sqrt{I^{\prime \prime}}$, the radical of $I^{\prime \prime}$, is the defining ideal for $\mathcal{A}^{\prime \prime}$. If $\mathcal{A}$ is generic and $n>2, I^{\prime \prime}$ is radical, so it is the defining ideal for $\mathcal{A}^{\prime \prime}$.

Since $I^{\prime \prime} \subseteq k\left[x_{2}, \ldots, x_{n}\right]$, we will consider $\mathcal{D}^{(m)}\left(I^{\prime \prime}\right)$ and, when applicable, $\Theta^{m}\left(I^{\prime \prime}\right)$ as $k\left[x_{2}, \ldots, x_{n}\right]$-submodules of $\mathcal{D}\left(k\left[x_{2}, \ldots, x_{n}\right]\right)$.

Let $j: \mathcal{D}^{(m)}\left(I^{\prime}\right) \longrightarrow \mathcal{D}^{(m)}(I)$ be left multiplication by $x_{1}$, and define $\pi$ by

$$
\pi: \sum f_{\beta}\left(x_{1}, \ldots, x_{n}\right) \partial^{\beta} \longmapsto \sum f_{\beta}\left(0, x_{2}, \ldots, x_{n}\right) \partial^{\beta}
$$

It is clear that if $\theta \in \mathcal{D}^{(m)}(R)$, then $\pi(\theta)$ is an operator of homogeneous order $m$. In [4] it was shown that the sequence

$$
0 \longrightarrow \operatorname{Der}\left(I^{\prime}\right) \stackrel{j}{\longrightarrow} \operatorname{Der}(I) \stackrel{\pi}{\longrightarrow} \operatorname{Der}\left(\sqrt{I^{\prime \prime}}\right),
$$

is exact. We will show that the corresponding sequence for homogeneous operators of higher order is also exact. However, in our proof that $\mathcal{D}^{(m)}(I)=\Theta^{m}(I)$, we will need a slightly different sequence. This is because when $n=2, \mathcal{A}^{\prime \prime}$ is the arrangement $\{0\} \subseteq k$, but $I^{\prime \prime}=\left\langle x_{2}^{r}\right\rangle$, so $I^{\prime \prime}$ is not the defining ideal for $\mathcal{A}^{\prime \prime}$ if $r>1$.

Lemma 5.20. Suppose $r \geq 1$, and that $I=\left\langle x_{1} p_{1} \cdots p_{r}\right\rangle$ defines a central arrangement. Then

$$
\pi\left(\mathcal{D}^{(m)}(I)\right) \subseteq \mathcal{D}^{(m)}\left(I^{\prime \prime}\right)
$$


and the sequence

$$
0 \longrightarrow \mathcal{D}^{(m)}\left(I^{\prime}\right) \stackrel{j}{\longrightarrow} \mathcal{D}^{(m)}(I) \stackrel{\pi}{\longrightarrow} \mathcal{D}^{(m)}\left(I^{\prime \prime}\right),
$$

of $k$-vector spaces, is exact.

Proof. Suppose $\theta \in \mathcal{D}^{(m)}(I)$. Then $\theta$ belongs to both $\mathcal{D}^{(m)}\left(\left\langle x_{1}\right\rangle\right)$ and $\mathcal{D}^{(m)}\left(I^{\prime}\right)$, by Theorem 3.5.

We know from Proposition 5.9 that $\mathcal{D}^{(m)}\left(\left\langle x_{1}\right\rangle\right)$ is generated as an $R$-module by $\left\{\partial^{\beta}|| \beta \mid=m\right.$ and $\left.\beta_{1}=0\right\}$ together with $\left\{x_{1} \partial^{\beta}|| \beta \mid=m\right.$ and $\left.\beta_{1}>0\right\}$. Thus any monomial in $\theta$ containing $\partial_{1}$ must also contain $x_{1}$. This shows that we may write

$$
\theta=x_{1} \theta_{1}+\theta_{2},
$$

where $\theta_{2}=\pi(\theta) \in \mathcal{D}^{(m)}\left(k\left[x_{2}, \ldots, x_{n}\right]\right)$.

Since $\theta \in \mathcal{D}^{(m)}\left(I^{\prime}\right)$ too, it follows in particular that for any $x^{\gamma}$ with $\gamma_{1}=0$ we have $\theta * x^{\gamma} p_{1} \cdots p_{r}=f p_{1} \cdots p_{r}$, for some $f \in R$. Each $p_{i}$ is of the form $p_{i}=a_{i} x_{1}+q_{i}$, for some $a_{i} \in k$, so

$$
p_{1} \cdots p_{r}=\left(a_{1} x_{1}+q_{1}\right) \cdots\left(a_{r} x_{1}+q_{r}\right)=x_{1} g+q_{1} \cdots q_{r},
$$

for some $g \in R$. Thus

$$
\begin{aligned}
\theta * x^{\gamma} p_{1} \cdots p_{r} & =\left(x_{1} \theta_{1}+\theta_{2}\right) * x^{\gamma} p_{1} \cdots p_{r} \\
& =x_{1} \theta_{1} * x^{\gamma} p_{1} \cdots p_{r}+\theta_{2} * x^{\gamma}\left(x_{1} g+q_{1} \cdots q_{r}\right) \\
& =x_{1} \theta_{1} * x^{\gamma} p_{1} \cdots p_{r}+x_{1} \theta_{2} * x^{\gamma} g+\theta_{2} * x^{\gamma} q_{1} \cdots q_{r},
\end{aligned}
$$

and

$$
f p_{1} \cdots p_{r}=x_{1} f g+f q_{1} \cdots q_{r}
$$

We may write $f=x_{1} f_{1}+f_{2}$, where no monomial in $f_{2}$ is divisible by $x_{1}$, so

$$
f p_{1} \cdots p_{r}=x_{1} f g+x_{1} f_{1} q_{1} \cdots q_{r}+f_{2} q_{1} \cdots q_{r} .
$$

Hence

$$
x_{1}\left(f g+f_{1} q_{1} \cdots q_{r}-\theta_{1} * x^{\gamma} p_{1} \cdots p_{r}-\theta_{2} * x^{\gamma} g\right)=\theta_{2} * x^{\gamma} q_{1} \cdots q_{r}-f_{2} q_{1} \cdots q_{r},
$$

where the right hand side is divisible by $x_{1}$ only if it is zero, so

$$
\theta_{2} * x^{\gamma} q_{1} \cdots q_{r}=f_{2} q_{1} \cdots q_{r} .
$$

We conclude that $\pi(\theta) \in \mathcal{D}^{(m)}\left(I^{\prime \prime}\right)$ whenever $\theta \in \mathcal{D}^{(m)}(I)$.

To see that the sequence (10) is exact, we begin by concluding that if $x_{1} \theta=0$, then $\theta=0$, so $j$ is an injection. It is clear that $\operatorname{Im} j \subseteq \operatorname{ker} \pi$. To show the reverse inclusion, take $\theta \in \operatorname{ker} \pi$. Then, by the above, $\theta=x_{1} \theta_{1}$, and $\theta \in \mathcal{D}^{(m)}\left(I^{\prime}\right)$. Hence, for any $f \in R$,

$$
x_{1} \theta_{1} * f p_{1} \cdots p_{r}=g p_{1} \cdots p_{r}
$$

for some $g \in R$. Since $x_{1}$ does not divide $p_{1} \cdots p_{r}$, it must divide $g$. Thus

$$
\theta_{1} * f p_{1} \cdots p_{r}=h p_{1} \cdots p_{r},
$$

where $x_{1} h=g$. It follows that $\theta_{1} \in \mathcal{D}^{(m)}\left(I^{\prime}\right)$, so $\theta \in x_{1} \mathcal{D}^{(m)}\left(I^{\prime}\right)=\operatorname{Im} j$. 
Again, the defining ideal for $\mathcal{A}^{\prime \prime}$ is $\sqrt{I^{\prime \prime}}$, which may differ from $I^{\prime \prime}$. However, as a corollary to the preceding lemma we have

Corollary 5.21. If $r \geq 1$, and $I=\left\langle x_{1} p_{1} \cdots p_{r}\right\rangle$ defines a central arrangement, then, also,

$$
\pi\left(\mathcal{D}^{(m)}(I)\right) \subseteq \mathcal{D}^{(m)}\left(\sqrt{I^{\prime \prime}}\right)
$$

so the generalization

$$
0 \longrightarrow \mathcal{D}^{(m)}\left(I^{\prime}\right) \stackrel{j}{\longrightarrow} \mathcal{D}^{(m)}(I) \stackrel{\pi}{\longrightarrow} \mathcal{D}^{(m)}\left(\sqrt{I^{\prime \prime}}\right),
$$

of the sequence (9), is exact for each $m \geq 0$.

Proof. It is enough to show that $\pi\left(\mathcal{D}^{(m)}(I)\right) \subseteq \mathcal{D}^{(m)}\left(\sqrt{I^{\prime \prime}}\right)$. The only way in which $I^{\prime \prime}$ may differ from $\sqrt{I^{\prime \prime}}$ is that $\pi$ maps different $p_{i}$ :s onto (nonconstant multiples of) the same $q_{j}$. Thus, after a renumbering, we may assume that $q_{1}, \ldots, q_{t}, t \leq r$, are pairwise relatively prime, and that $I^{\prime \prime}=\left\langle q_{1}^{l_{1}} \cdots q_{t}^{l_{t}}\right\rangle$, where $\pi\left(p_{i}\right)=q_{i}$, for $i=1, \ldots, t$, each $l_{j} \geq 1$, and $l_{1}+\ldots+l_{t}=r$. Write $J=$ $\left\langle x_{1} p_{1} \cdots p_{t}\right\rangle$ and $J^{\prime \prime}=\left\langle q_{1} \cdots q_{t}\right\rangle=\sqrt{I^{\prime \prime}}$. Then

$$
\mathcal{D}^{(m)}(I)=\mathcal{D}^{(m)}(J) \cap \mathcal{D}^{(m)}\left(\left\langle p_{t+1} \cdots p_{r}\right\rangle\right) \subseteq \mathcal{D}^{(m)}(J)
$$

by Theorem 3.5 , and $\pi\left(\mathcal{D}^{(m)}(J)\right) \subseteq \mathcal{D}^{(m)}\left(J^{\prime \prime}\right)$, by Lemma 5.20.

If the arrangement defined by $I$ is not generic, $\pi$ need not be onto.

Example 5.22. Let $R=k[x, y, z]$ and let $\mathcal{A}$ be the arrangement in $k^{3}$ defined by $I=\langle x y z(x+y)(x+z)\rangle \subseteq R$. Then $\mathcal{A}$ is not generic, by Example 4.5 (iii). Here $I^{\prime \prime}=\left\langle y^{2} z^{2}\right\rangle$, so $\sqrt{I^{\prime \prime}}=\langle y z\rangle$. Let $S=k[y, z]$. Using Proposition 3.2, we see that

$$
\operatorname{Der}\left(I^{\prime \prime}\right)=\operatorname{Der}\left(\sqrt{I^{\prime \prime}}\right)=S y \partial_{y}+S z \partial_{z} .
$$

On the other hand, by [4, Theorem 4.23], Der $(I)$ is generated by $\varepsilon, y(x+y) \partial_{y}$, and $z(x+z) \partial_{z}$, as an $R$-module, so

$$
\pi(\operatorname{Der}(I))=S\left(y \partial_{y}+z \partial_{z}\right)+S y^{2} \partial_{y}+S z^{2} \partial_{z} \subsetneq \operatorname{Der}\left(I^{\prime \prime}\right)=\operatorname{Der}\left(\sqrt{I^{\prime \prime}}\right) .
$$

Thus $\pi$ is not onto if $m=1$.

The fact that $\pi: \mathcal{D}^{(m)}(I) \longrightarrow \mathcal{D}^{(m)}\left(I^{\prime \prime}\right)$ is onto whenever the arrangement defined by $I$ is generic, is the content of Corollary 5.26 .

We will now see what $\mathcal{D}^{(m)}\left(I^{\prime \prime}\right)$ is when $n=2$.

Lemma 5.23. If $r \geq 1$, and $I=\left\langle x_{1} p_{1} \cdots p_{r}\right\rangle$ defines a central arrangement in $k^{2}$, then $I^{\prime \prime}=\left\langle x_{2}^{r}\right\rangle$ and

$$
\mathcal{D}^{(m)}\left(I^{\prime \prime}\right)=k\left[x_{2}\right] x_{2}^{\min \{r, m\}} \partial_{2}^{m} .
$$

Proof. Any element in $\mathcal{D}^{(m)}\left(k\left[x_{2}\right]\right)$ is of the form $f \partial_{2}^{m}$, where $f \in k\left[x_{2}\right]$. By Proposition $3.2, f \partial_{2}^{m}$ preserves $\left\langle x_{2}^{r}\right\rangle$ if and only if

$$
\left\langle x_{2}^{r}\right\rangle \ni f \partial_{2}^{m} * x_{2}^{r+j}= \begin{cases}\frac{(r+j) !}{(r+j-m) !} f x_{2}^{r+j-m} & \text { if } m \leq r+j \\ 0 & \text { if } m>r+j\end{cases}
$$

for every $j \leq m-1$. From this, the lemma follows easily. 
Lemma 5.24. If $r \geq 1$, and $I=\left\langle x_{1} p_{1} \cdots p_{r}\right\rangle$ defines a generic arrangement, then the restriction of $\pi$ to $\Theta^{m}(I)$ is a surjection onto $\Theta^{m}\left(I^{\prime \prime}\right)$ when $n>2$, and a surjection onto $\mathcal{D}^{(m)}\left(I^{\prime \prime}\right)$ when $n=2$. In both cases the restriction of $j$ to $\Theta^{m}\left(I^{\prime}\right)$ is an injection into $\Theta^{m}(I)$.

Thus, when $n>2$, the sequence

$$
0 \longrightarrow \Theta^{m}\left(I^{\prime}\right) \stackrel{j}{\longrightarrow} \Theta^{m}(I) \stackrel{\pi}{\longrightarrow} \Theta^{m}\left(I^{\prime \prime}\right) \longrightarrow 0,
$$

is exact, except possibly at $\Theta^{m}(I)$, and when $n=2$, the same thing holds for the sequence

$$
0 \longrightarrow \Theta^{m}\left(I^{\prime}\right) \stackrel{j}{\longrightarrow} \Theta^{m}(I) \stackrel{\pi}{\longrightarrow} \mathcal{D}^{(m)}\left(I^{\prime \prime}\right) \longrightarrow 0 .
$$

As in Lemma 5.20, these are sequences of $k$-vector spaces.

Proof. Let $M=\left\{\delta_{1}, \ldots, \delta_{s}\right\}$ be the set associated to $x_{1}, p_{1}, \ldots, p_{r}$ according to Lemma 5.13. Then $M$ contains a basis for each intersection of $V_{i}$ :s; in particular for each intersection of $V_{i}$ :s corresponding to $p_{1}, \ldots, p_{r}$. Thus the set $M^{\prime}$ associated to $p_{1}, \ldots, p_{r}$ according to Lemma 5.13 may be taken as a subset of $M$. From this it follows that

$$
\Theta^{m}\left(I^{\prime}\right)=\sum R P_{\alpha}^{\prime} \delta^{\alpha}+R \varepsilon_{m}
$$

where the sum ranges over those $\alpha$ such that $\alpha_{i} \neq 0$ only if $\delta_{i}$ belongs to $M^{\prime}$, and the factors in $P_{\alpha}^{\prime}$ are chosen among $p_{1}, \ldots, p_{r}$. It follows that

$$
j\left(\Theta^{m}\left(I^{\prime}\right)\right)=x_{1} \Theta^{m}\left(I^{\prime}\right) \subseteq \Theta^{m}(I) .
$$

Now assume $n>2$. Number the $V_{i}$ : s so that $V_{0}$ corresponds to $x_{1}$, and $V_{i}$ corresponds to $p_{i}$, for $i=1, \ldots, r$. Note that since the derivations in $V_{0}$ annihilate $x_{1}$, they all have $\partial_{1}$-coefficient equal to zero. Let $W=\sum_{j=2}^{n} k \partial_{j}$, and let $W_{i}$ be the subspace of $W$ generating the module of derivations annihilating $q_{i}, i=1, \ldots, r$. Then

$$
W_{i_{1}} \cap \ldots \cap W_{i_{t}}=V_{0} \cap V_{i_{1}} \cap \ldots \cap V_{i_{t}} \subseteq W
$$

by the isomorphism between $V_{H}$ and $H$. Let $M^{\prime \prime}$ be the subset of $M$ consisting of the $\delta_{i}$ :s with $\partial_{1}$-coefficient equal to zero. From the above, it follows that $M^{\prime \prime}$ is the set associated to $q_{1}, \ldots, q_{r}$ according to Lemma 5.13. Thus, letting $Q_{\alpha}\left(x_{2}, \ldots, x_{n}\right)=P_{\alpha}\left(0, x_{2}, \ldots, x_{n}\right)$, we see that the generators for $\Theta^{m}\left(I^{\prime \prime}\right)$ as $k\left[x_{2}, \ldots, x_{n}\right]$-module are

$$
\left\{Q_{\alpha} \delta^{\alpha}|| \alpha \mid=m\right\} \cup\left\{\epsilon_{m}\right\}
$$

where the $\delta_{i}$ :s with $\alpha_{i} \neq 0$ are chosen from $M^{\prime \prime}$, and $\epsilon_{m}=\pi\left(\varepsilon_{m}\right)$ is the operator corresponding to $\varepsilon_{m}$ in $\mathcal{D}\left(k\left[x_{2}, \ldots, x_{n}\right]\right)$. Thus any element in $\Theta^{m}\left(I^{\prime \prime}\right)$ is the image, under $\pi$, of some operator in $\Theta^{m}(I)$.

On the other hand, for any $R$-module generator $P_{\alpha} \delta^{\alpha}$ of $\Theta^{m}(I)$, in which some $\delta_{i}$ with $\alpha_{i} \neq 0$ does not belong to $M^{\prime \prime}, x_{1}$ must be a factor in $P_{\alpha}$, so $\pi\left(x^{\beta} P_{\alpha} \delta^{\alpha}\right)=0$, for any monomial $x^{\beta} \in R$. Whence $\pi$ maps $\Theta^{m}(I)$ onto $\Theta^{m}\left(I^{\prime \prime}\right)$.

When $n=2$, the set $M$ from Lemma 5.13 will consist of $r+1$ derivations, each one annihilating precisely one of $x_{1}, p_{1}, \ldots, p_{r}$. Thus all $R$-module generators 
of $\Theta^{m}(I)$ of the form $P_{\alpha} \delta^{\alpha}$, except for $p_{1} \cdots p_{r} \partial_{2}^{m}$, will have $x_{1}$ as a factor in $P_{\alpha}$. Hence the image of $\Theta^{m}(I)$ under $\pi$ is generated, as a $k\left[x_{2}\right]$-module, by

$$
\pi\left(\varepsilon_{m}\right)=x_{2}^{m} \partial_{2}^{m}
$$

and

$$
\pi\left(p_{1} \cdots p_{r} \partial_{2}^{m}\right)=a x_{2}^{r} \partial_{2}^{m},
$$

where $a$ is some nonzero constant. This means that

$$
\pi\left(\Theta^{m}(I)\right)=k\left[x_{2}\right] x_{2}^{\min \{r, m\}} \partial_{2}^{m},
$$

which, by Lemma 5.23, finishes the proof.

We can now show

Proposition 5.25. Suppose that $I=\left\langle p_{1} \cdots p_{r}\right\rangle$ defines a generic arrangement. Then

$$
\mathcal{D}^{(m)}(I)=\Theta^{m}(I),
$$

as R-modules.

Proof. By Proposition 5.18 it is enough to show that

$$
\mathcal{D}^{(m)}(I) \subseteq \Theta^{m}(I),
$$

as $k$-vector spaces. To do so we proceed by double induction, on the number $r \geq 1$ of hyperplanes, and on the dimension $n \geq 2$ of the ambient space of the arrangement.

The case $r=1$ follows from Proposition 5.9. The case $n=2$ will be dealt with in a while.

Thus suppose that $r \geq 1$ and $n \geq 2$, and that the inclusion (12) is true for $r$ and $n$, and for $r$ and $n+1$, respectively. Suppose that $p_{1}, \ldots, p_{r+1}$ are linear forms in $k\left[x_{1}, \ldots, x_{n+1}\right]$ defining a generic arrangement. We may assume, after a linear change of coordinates, that $p_{r+1}=x_{1}$. Thus, letting $I=\left\langle x_{1} p_{1} \cdots p_{r}\right\rangle$, it follows from Lemmas 5.20 and 5.24 that the rows in the commutative diagram

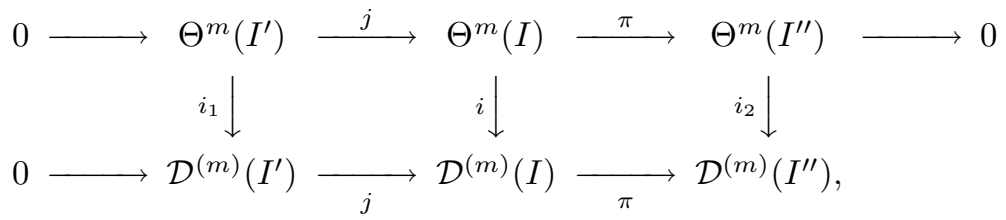

of $k$-vector spaces are exact, except possibly at $\Theta^{m}(I)$. Here $i, i_{1}$, and $i_{2}$ are inclusions, by Proposition 5.18.

By the induction hypothesis, $i_{1}$ and $i_{2}$ are actually identity maps. Thus assume that $\theta \in \mathcal{D}^{(m)}(I)$. Then $\pi(\theta) \in \mathcal{D}^{(m)}\left(I^{\prime \prime}\right)=\pi\left(\Theta^{m}(I)\right)$. Hence there exists $\psi \in \Theta^{m}(I) \subseteq \mathcal{D}^{(m)}(I)$ such that $\pi(\psi)=\pi(\theta)$. Then

$$
\theta-\psi \in \operatorname{ker} \pi=\operatorname{Im} j=x_{1} \mathcal{D}^{(m)}\left(I^{\prime}\right)=x_{1} \Theta^{m}\left(I^{\prime}\right) \subseteq \Theta^{m}(I) .
$$

This implies that $\theta \in \Theta^{m}(I)$, which shows that $\mathcal{D}^{(m)}(I) \subseteq \Theta^{m}(I)$. 
To show the proposition for $n=2$, we use induction on the number of hyperplanes. Proceeding as above we get the commutative diagram

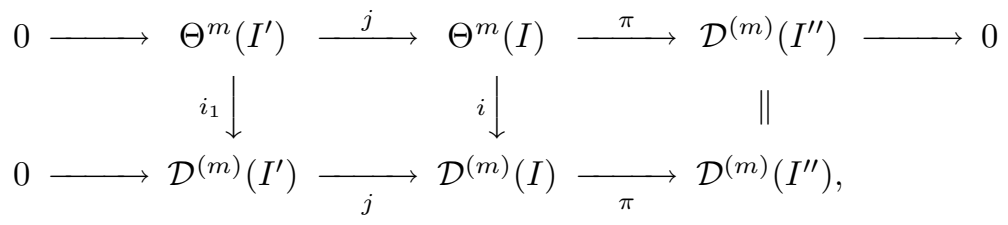

where $i_{1}$, by the induction hypothesis, is the identity map. The same diagram chase as above concludes the proof.

Corollary 5.26. If $r \geq 1$, and $I=\left\langle x_{1} p_{1} \cdots p_{r}\right\rangle$ defines a generic arrangement, then the sequence of $k$-vector spaces

$$
0 \longrightarrow \mathcal{D}^{(m)}\left(I^{\prime}\right) \stackrel{j}{\longrightarrow} \mathcal{D}^{(m)}(I) \stackrel{\pi}{\longrightarrow} \mathcal{D}^{(m)}\left(I^{\prime \prime}\right) \longrightarrow 0
$$

is exact for each $m \geq 0$.

In Proposition 5.9 we did not need $\varepsilon_{m}$ as a generator for $\mathcal{D}^{(m)}(I)$. This remains true for all $r \leq n$.

Lemma 5.27. If $r \leq n$ and $p_{1}, \ldots, p_{r}$ define a generic arrangement, then

$$
\varepsilon_{m} \in \sum_{|\alpha|=m}\left(I:\left(I: P^{\alpha}\right)\right) \delta^{\alpha} .
$$

Proof. It is enough to show how $\varepsilon$ may be expressed as a linear combination of $P_{i} \delta_{i}$, since the homogeneous component of highest order of a power of such an expression must be a linear combination of the $P_{\alpha} \delta^{\alpha},|\alpha|=m$.

If $r=n$, not only is $M=\left\{\delta_{1}, \ldots, \delta_{n}\right\}$ from Lemma 5.13 a basis for $V$, but $\left\{p_{1}, \ldots, p_{n}\right\}$ is a basis for $\sum k x_{i}$. Thus

$$
x_{l}=\sum_{j} a_{l j} p_{j},
$$

for some $a_{l j} \in k$, and by the proposition above, $\operatorname{Der}\left(\left\langle p_{1} \cdots p_{n}\right\rangle\right)$ is generated by $p_{1} \delta_{1}, \ldots, p_{n} \delta_{n}$ together with $\varepsilon$.

Let

$$
\theta=\sum_{i} \frac{p_{i} \delta_{i}}{\delta_{i} * p_{i}} \in \operatorname{Der}\left(\left\langle p_{1} \cdots p_{n}\right\rangle\right) .
$$

Then

$$
\theta * x_{l}=\left(\sum_{i} \frac{p_{i} \delta_{i}}{\delta_{i} * p_{i}}\right) *\left(\sum_{j} a_{l j} p_{j}\right)=\sum_{j} a_{l j} \frac{p_{j} \delta_{j} * p_{j}}{\delta_{j} * p_{j}}=\sum_{j} a_{l j} p_{j}=x_{l},
$$

for $l=1, \ldots, n$, so $\theta=\varepsilon$, by Lemma 2.4 .

If $r<n$ there are linear forms $p_{r+1}, \ldots, p_{n} \in R$ such that $\delta_{i}$ annihilates all $p_{j}$ :s, except $p_{i}$, for $i=r+1, \ldots, n$, and such that $\left\{p_{1}, \ldots, p_{n}\right\}$ is a basis for $\sum k x_{i}$. Then the same result as above holds.

We are now ready to formulate the main result of this section. 
Theorem 5.28. Suppose $I=\left\langle p_{1} \cdots p_{r}\right\rangle$ defines a generic arrangement. Let

$$
D=\left\{P_{1} \delta_{1}, \ldots, P_{s} \delta_{s}\right\}
$$

be the set associated to I according to Definition 5.14. Then

$$
\mathcal{D}(I)=\bigoplus_{m \geq 0}\left(\sum_{|\alpha|=m}\left(I:\left(I: P^{\alpha}\right)\right) \delta^{\alpha}+R \varepsilon_{m}\right),
$$

as an $R$-module, where $R \varepsilon_{m} \subseteq \sum_{|\alpha|=m}\left(I:\left(I: P^{\alpha}\right)\right) \delta^{\alpha}$, if $r \leq n$.

Proof. This follows immediately from Proposition 5.3, Proposition 5.25, and from Lemma 5.27, together.

Let us write $\overline{\varepsilon_{m}}$ for $\sum_{|\alpha|=m} \frac{m !}{\alpha !} x^{\alpha} \partial^{\alpha}$, the operator in $\mathcal{D}(R / I)$ induced by $\varepsilon_{m}$, as in Section 2.2. Then, by Theorem 2.5 we get

Corollary 5.29. Suppose $A$ is the coordinate ring of a generic arrangement, and let

$$
D=\left\{P_{1} \delta_{1}, \ldots, P_{s} \delta_{s}\right\}
$$

be the set associated to the defining ideal $I=\left\langle p_{1} \cdots p_{r}\right\rangle$, according to Definition 5.14. Then

$$
\mathcal{D}(A)=\bigoplus_{m \geq 0}\left(\sum_{|\alpha|=m} \overline{\left(I:\left(I: P^{\alpha}\right)\right)} \delta^{\alpha}+A \overline{\varepsilon_{m}}\right),
$$

as an $A$-module, where $A \overline{\varepsilon_{m}} \subseteq \sum_{|\alpha|=m} \overline{\left(I:\left(I: P^{\alpha}\right)\right)} \delta^{\alpha}$, if $r \leq n$.

Remark. It is clear that $\overline{\left(I:\left(I: P^{\alpha}\right)\right)} \neq 0$ if and only if $P^{\alpha} \notin I$. This is the case if and only if there is some $p_{i}$ with $\delta_{j} * p_{i}=0$ for all $j$ with $\alpha_{j} \neq 0$, i.e., if and only if $\delta^{\alpha} \in V_{i}^{|\alpha|}$, for some $i$.

The case $n=2$ is special, since by Example 4.5 (ii), any central arrangement in the plane is generic. We therefore state this case explicitly in the following corollary to Theorem 5.28.

Corollary 5.30. Suppose that $I=\left\langle p_{1} \cdots p_{r}\right\rangle \subseteq k[x, y]=R$ defines a central arrangement. Let

$$
P_{i}=p_{1} \cdots \widehat{p}_{i} \cdots p_{r}
$$

with $P_{1}=1$ if $r=1$, and

$$
\delta_{i}= \begin{cases}\partial_{y} & \text { if } p_{i}=a x, a \in k \backslash\{0\}, \\ \partial_{x}+a_{i} \partial_{y} & \text { if } p_{i}=a\left(y-a_{i} x\right), a \in k \backslash\{0\},\end{cases}
$$

for $i=1, \ldots, r$. Then

$$
\mathcal{D}(I)=R+\bigoplus_{m \geq 1}\left(R P_{1} \delta_{1}^{m}+\ldots+R P_{r} \delta_{r}^{m}+R \varepsilon_{m}\right)+I \mathcal{D}(R),
$$

as an $R$-module. 
Proof. If $r>1$, the set $D$ associated to $I$ according to Definition 5.14 will be

$$
D=\left\{P_{1} \delta_{1}, \ldots, P_{r} \delta_{r}\right\}
$$

with $P_{i}$ and $\delta_{i}$ as above. If $r=1$, let $P_{1}$ and $\delta_{1}$ be as above. Let $P_{2}=p_{1}$ and let $\delta_{2}$ be any derivation in $k \partial_{x}+k \partial_{y}$, which is not a multiple of $\delta_{1}$. Then

$$
D=\left\{P_{1} \delta_{1}, P_{2} \delta_{2}\right\}
$$

Finally, for a product, $\delta^{\alpha}$, of distinct $\delta_{i}$ :s, we have $P_{\alpha}=p_{1} \cdots p_{r}$.

Corollary 5.31. Suppose that $A$ is the coordinate ring of a central arrangement in the plane, defined by $I=\left\langle p_{1} \cdots p_{r}\right\rangle \subseteq k[x, y]$. Then, with notation as in Corollary 5.30,

$$
\mathcal{D}(A)=A+\bigoplus_{m \geq 1}\left(A \overline{P_{1}} \delta_{1}^{m}+\ldots+A \overline{P_{r}} \delta_{r}^{m}+A \overline{\varepsilon_{m}}\right)
$$

as an A-module.

Observe that, if $k$ is algebraically closed, the result in Corollaries 5.30 and 5.31 are true for any principal ideal which is homogeneous and radical.

We have only defined $\Theta^{m}(I)$ for the case when $I$ defines a generic arrangement. It is of course possible to do the same for any ideal defining a central arrangement:

Suppose $I=\left\langle p_{1} \cdots p_{r}\right\rangle$ defines a central arrangement.

Let $M=\left\{\delta_{1}, \ldots, \delta_{s}\right\}$ be a subset of $V$ containing a basis for each intersection of $V_{i}: \mathrm{s}$.

Let $D=\left\{P_{1} \delta_{1}, \ldots, P_{s} \delta_{s}\right\}$, where $P_{i}$ is the product of the $p_{j}:$ s which are not annihilated by $\delta_{i}$.

Define $\Theta^{m}(I)=\sum_{|\alpha|=m}\left(I:\left(I: P^{\alpha}\right)\right) \delta^{\alpha}+R \varepsilon_{m}$.

Then, as in Proposition 5.18, and with the same proof, we have

$$
\Theta^{m}(I) \subseteq \mathcal{D}^{(m)}(I)
$$

We will end this section with an example showing that the opposite inclusion need not hold if the arrangement defined by $I$ is not generic. Hence Theorem 5.28 is not true for nongeneric arrangements.

Example 5.32. Let $I=\langle x y(x-y)\rangle \subseteq k[x, y, z]=R$. Let $\mathcal{A}$ be the central arrangement of three planes in $k^{3}$, defined by $I$. Then $\mathcal{A}$ is nongeneric, by Example 4.5 (iii).

We have that $\varepsilon, \partial_{z}$, and $z \partial_{z}^{2}$ all preserve $I$, so

$$
\left(x \partial_{x}+y \partial_{y}\right) \partial_{z}=\varepsilon \partial_{z}-z \partial_{z}^{2} \in \mathcal{D}^{(2)}(I)
$$

To find $\Theta^{2}(I)$, let $x=p_{1}, y=p_{2}$, and $x-y=p_{3}$. Then

$$
V_{1} \cap V_{2} \cap V_{3}=V_{1} \cap V_{2}=V_{1} \cap V_{3}=V_{2} \cap V_{3}=k \partial_{z} .
$$


Extending this to bases for the $V_{i}$ :s, we may choose

$$
\begin{aligned}
& V_{1}=k \partial_{y}+k \partial_{z}, \\
& V_{2}=k \partial_{x}+k \partial_{z}, \\
& V_{3}=k\left(\partial_{x}+\partial_{y}\right)+k \partial_{z} .
\end{aligned}
$$

Thus $M=\left\{\partial_{x}, \partial_{x}+\partial_{y}, \partial_{y}, \partial_{z}\right\}$ has the property of containing a basis for each intersection of $V_{i}: s$, as well as for $V$, so

$$
D=\left\{x(x-y) \partial_{x}, x y\left(\partial_{x}+\partial_{y}\right), y(x-y) \partial_{y}, \partial_{z}\right\} .
$$

Hence, after removing unnecessary generators,

$$
\begin{aligned}
\Theta^{2}(I)= & R x(x-y) \partial_{x}^{2}+R x y\left(\partial_{x}+\partial_{y}\right)^{2}+R y(x-y) \partial_{y}^{2} \\
& +R x(x-y) \partial_{x} \partial_{z}+R x y\left(\partial_{x}+\partial_{y}\right) \partial_{z}+R y(x-y) \partial_{y} \partial_{z} \\
& +R \partial_{z}^{2}+R \varepsilon_{2} .
\end{aligned}
$$

All the polynomial coefficients in these generators, except for $\partial_{z}^{2}$, have degree two, but the polynomial coefficients in the element $\left(x \partial_{x}+y \partial_{y}\right) \partial_{z}$ of $\mathcal{D}^{(2)}(I)$, only have degree one, so it cannot possibly belong to $\Theta^{2}(I)$. Whence

$$
\Theta^{2}(I) \subsetneq \mathcal{D}^{(2)}(I)
$$

in this case.

\subsubsection{Algebra Generators}

As pointed out earlier, Nakai's conjecture holds for the coordinate ring of an affine arrangement. Thus, in particular, if $A$ is the coordinate ring of a generic arrangement of more than one hyperplane, then $\mathcal{D}(A) \neq \Delta(A)$. We will use Theorem 5.28 to find finitely many $k$-algebra generators for $\mathcal{D}(I)$. Then, by Theorem 2.5, it follows that, although not generated by $A$ and derivations, $\mathcal{D}(A)$ is still finitely generated as a $k$-algebra.

Lemma 5.33. Let $p_{1}, \ldots, p_{t}, t \geq 1$, be polynomials in $R$ of degree one and let $\delta \in \operatorname{Der}(R)$ be a derivation with coefficients in $k$. Then

$$
\sum_{i=0}^{t}(-1)^{i}\left(\begin{array}{l}
t \\
i
\end{array}\right) \delta^{2 t+1-i} p_{1} \cdots p_{t} \delta^{i}=\left(\delta * p_{1}\right) \cdots\left(\delta * p_{t}\right) t ! \delta^{t+1} .
$$

Proof. We will prove this by induction on $t$. If $t=1$, then

$$
\begin{aligned}
\sum_{i=0}^{1}(-1)^{i}\left(\begin{array}{l}
1 \\
i
\end{array}\right) \delta^{3-i} p_{1} \delta^{i} & =\delta^{3} p_{1}-\delta^{2} p_{1} \delta=\delta^{2}\left(\delta p_{1}-p_{1} \delta\right) \\
& =\delta^{2}\left(\delta * p_{1}\right)=\left(\delta * p_{1}\right) \delta^{2} .
\end{aligned}
$$

Now assume that the lemma is true for some $t \geq 1$. Agreeing that $\left(\begin{array}{c}t \\ t+1\end{array}\right)=$ 
$\left(\begin{array}{c}t \\ -1\end{array}\right)=0$, we get

$$
\begin{aligned}
& \sum_{i=0}^{t+1}(-1)^{i}\left(\begin{array}{c}
t+1 \\
i
\end{array}\right) \delta^{2 t+3-i} p_{1} \cdots p_{t+1} \delta^{i} \\
& =\sum_{i=0}^{t+1}(-1)^{i}\left(\left(\begin{array}{c}
t \\
i
\end{array}\right)+\left(\begin{array}{c}
t \\
i-1
\end{array}\right)\right) \delta^{2 t+3-i} p_{1} \cdots p_{t+1} \delta^{i} \\
& =\sum_{i=0}^{t}(-1)^{i}\left(\begin{array}{c}
t \\
i
\end{array}\right) \delta^{2 t+3-i} p_{1} \cdots p_{t+1} \delta^{i}-\sum_{i=0}^{t}(-1)^{i}\left(\begin{array}{c}
t \\
i
\end{array}\right) \delta^{2 t+2-i} p_{1} \cdots p_{t+1} \delta^{1+i} \\
& =\sum_{i=0}^{t}(-1)^{i}\left(\begin{array}{c}
t \\
i
\end{array}\right) \delta^{2 t+2-i}\left(p_{1} \cdots p_{t+1} \delta+\sum_{j=1}^{t+1}\left(\delta * p_{j}\right) p_{1} \cdots \widehat{p_{j}} \cdots p_{t+1}\right) \delta^{i} \\
& \quad-\sum_{i=0}^{t}(-1)^{i}\left(\begin{array}{l}
t \\
i
\end{array}\right) \delta^{2 t+2-i} p_{1} \cdots p_{t+1} \delta^{1+i} \\
& =\sum_{i=0}^{t}(-1)^{i}\left(\begin{array}{c}
t \\
i
\end{array}\right) \delta^{2 t+2-i} \sum_{j=1}^{t+1}\left(\delta * p_{j}\right) p_{1} \cdots \widehat{p_{j}} \cdots p_{t+1} \delta^{i} \\
& =\sum_{j=1}^{t+1}\left(\delta * p_{j}\right) \delta\left(\sum_{i=0}^{t}(-1)^{i}\left(\begin{array}{l}
t \\
i
\end{array}\right) \delta^{2 t+1-i} p_{1} \cdots \widehat{p_{j}} \cdots p_{t+1} \delta^{i}\right)
\end{aligned}
$$

(by the induction hypothesis)

$$
\begin{aligned}
& =\sum_{j=1}^{t+1}\left(\delta * p_{j}\right) \delta\left(\delta * p_{1}\right) \cdots\left(\widehat{\delta * p_{j}}\right) \cdots\left(\delta * p_{t+1}\right) t ! \delta^{t+1} \\
& =\sum_{j=1}^{t+1}\left(\delta * p_{1}\right) \cdots\left(\delta * p_{t+1}\right) t ! \delta^{t+2} \\
& =\left(\delta * p_{1}\right) \cdots\left(\delta * p_{t+1}\right)(t+1) ! \delta^{t+2}
\end{aligned}
$$

Lemma 5.34. Let $p_{1}, \ldots, p_{t}, t \geq 1$, be polynomials in $R$ of degree one and let $\delta \in \operatorname{Der}(R)$ be a derivation with coefficients in $k$ such that $\delta * p_{j} \neq 0$, for $j=1, \ldots, t$. Then, for any $m>0$, we may write $p_{1} \cdots p_{t} \delta^{m}$ as a $k$-linear combination of products of $p_{1} \cdots p_{t} \delta^{l}$ with $1 \leq l \leq 2 t+1$.

Proof. If $m \leq 2 t+1$ there is nothing to prove, so assume that $m>2 t+1$. Then, by Lemma 5.33 , we have

$$
\begin{aligned}
\sum_{i=0}^{t} & (-1)^{i}\left(\begin{array}{c}
t \\
i
\end{array}\right) p_{1} \cdots p_{t} \delta^{m-1-i} p_{1} \cdots p_{t} \delta^{t+1+i} \\
& =p_{1} \cdots p_{t} \delta^{m-2 t-2}\left(\sum_{i=0}^{t}(-1)^{i}\left(\begin{array}{c}
t \\
i
\end{array}\right) \delta^{2 t+1-i} p_{1} \cdots p_{t} \delta^{i}\right) \delta^{t+1} \\
& =p_{1} \cdots p_{t} \delta^{m-2 t-2}\left(\delta * p_{1}\right) \cdots\left(\delta * p_{t}\right) t ! \delta^{t+1} \delta^{t+1} \\
& =\left(\delta * p_{1}\right) \cdots\left(\delta * p_{t}\right) t ! p_{1} \cdots p_{t} \delta^{m},
\end{aligned}
$$


i.e.,

$$
p_{1} \cdots p_{t} \delta^{m}=\sum_{i=0}^{t} a_{i} p_{1} \cdots p_{t} \delta^{m-1-i} p_{1} \cdots p_{t} \delta^{t+1+i},
$$

where $a_{i} \in k \backslash\{0\}$, for $i=0, \ldots, t$. We observe that in each term in the sum (13) we have

$$
1 \leq m-1-i<m \quad \text { and } \quad 1 \leq t+1+i \leq 2 t+1 .
$$

Hence the lemma follows, by induction on $m$.

Example 5.11 (continued from page 16). Finding generators for $\mathcal{D}(A)$ as a $k$-algebra, where $A$ is the coordinate ring of three distinct lines in the plane, we have, using the same notation as before,

(i) Three parallel lines.

By Example 5.10, $\mathcal{D}(A)$ is generated by $A$ and a derivation $\delta_{1}$, with coefficients in $k$, which annihilates all of $p_{1}, p_{2}$, and $p_{3}$.

In cases (ii), (iii), and (iv) it is clear that, as in the proof of Corollary 5.30, any product of two generators of the form $\bar{p} \delta_{i}$, with different $i: s$, equals zero. Thus, using Lemma 5.34, we get:

(ii) Two parallel lines intersecting a third.

In this case $\mathcal{D}(A)$ is generated by $A$ and

$$
\left\{\overline{p_{3}} \delta_{1}^{m} \mid m=1,2,3\right\} \cup\left\{\overline{p_{1} p_{2}} \delta_{2}^{m} \mid m=1,2,3,4,5\right\} .
$$

(iii) Three nonparallel lines with no common point of intersection.

Here $\mathcal{D}(A)$ is generated by

$$
\left\{\overline{x(x-y+1)} \partial_{x}^{m}, \overline{x y}\left(\partial_{x}+\partial_{y}\right)^{m}, \overline{y(x-y+1)} \partial_{y}^{m} \mid m=1,2,3,4,5\right\},
$$

together with A.

(iv) Three lines intersecting in one point.

This case follows from Proposition 5.38.

Theorem 5.35. Suppose that $I=\left\langle p_{1} \cdots p_{r}\right\rangle$ defines a generic arrangement. Then $\mathcal{D}(I)$ is finitely generated as a $k$-algebra. More precisely:

If $r \leq n$, then $\mathcal{D}(I)$ is generated by

$$
\left\{x_{1}, \ldots, x_{n}, \delta_{r+1}, \ldots, \delta_{n}\right\} \cup\left\{p_{i} \delta_{i}, p_{i} \delta_{i}^{2}, p_{i} \delta_{i}^{3} \mid i=1, \ldots, r\right\},
$$

where $\left\{p_{1} \delta_{1}, \ldots, p_{r} \delta_{r}, \delta_{r+1}, \ldots, \delta_{n}\right\}$ is the set $D$ from Definition 5.14.

If $r>n$, then $\mathcal{D}(I)$ is generated by

$$
\left\{x_{1}, \ldots, x_{n}, \varepsilon\right\} \cup\left\{P_{\alpha} \delta^{\alpha} \mid \alpha_{i} \leq 2(r-n)+3 \text { for } i=1, \ldots, s\right\},
$$

with $P_{\alpha}$ as in Definition 5.16 for each $\alpha \in \mathbb{N}^{s}$. 
Proof. First suppose that $r \leq n$. Then every $R$-module generator of $\mathcal{D}(I)$ of order $\geq 1$ is of the form $P_{\alpha} \delta^{\alpha}$, as defined in 5.16. However, the elements in $D$ commute with each other, so it is enough to show that for $i=1, \ldots, r$ we may write $p_{i} \delta_{i}^{m}$ as a sum of products of operators of lower order, if $m$ is large enough. It follows from Lemma 5.34 that this is possible if $m>3$.

Hence $\left\{x_{1}, \ldots, x_{n}, \delta_{r+1}, \ldots, \delta_{n}\right\} \cup\left\{p_{i} \delta_{i}, p_{i} \delta_{i}^{2}, p_{i} \delta_{i}^{3} \mid i=1, \ldots, r\right\}$ generate $\mathcal{D}(I)$ as a $k$-algebra.

Now suppose that $r>n$. The $R$-module generators $\varepsilon_{m}$ are $k$-linear combinations of powers of $\varepsilon$, so it remains to show that each $R$-module generator of the form $P_{\alpha} \delta^{\alpha}$ may be expressed using lower order operators, if $|\alpha|$ is large enough. In fact, we will show that any operator of the form $P_{\alpha} \delta^{\alpha}$ may be written as a $k$-linear combination of products of operators of the form $P_{\beta} \delta^{\beta}$ with $\beta_{i} \leq 2(r-n)+3$, for $i=1, \ldots, s$, and such that $\beta_{i}=0$ if $\alpha_{i}=0$.

Therefore assume that $P_{\alpha} \delta^{\alpha}$ is an $R$-module generator of $\mathcal{D}(I)$ with $\alpha_{i}>$ $2(r-n)+3$ for some $i$.

The derivations $\delta_{1}, \ldots, \delta_{s}$ all commute with each other, so any product $\delta^{\alpha}=$ $\delta_{1}^{\alpha_{1}} \cdots \delta_{s}^{\alpha_{s}}$ may be rearranged as $\delta^{\alpha}=\delta_{i}^{\alpha_{i}} \delta_{1}^{\alpha_{1}} \cdots \widehat{\delta_{i}^{\alpha_{i}}} \cdots \delta_{s}^{\alpha_{s}}=\delta_{i}^{\alpha_{i}} \delta^{\alpha-\alpha_{i} e_{i}}$.

Furthermore, $P_{\alpha}=P^{\prime} P_{i}$, where $P^{\prime}$ is the product of those $p_{j}$ :s in $P_{\alpha}$ which are annihilated by $\delta_{i}$, or $P^{\prime}=1$ if no $p_{j}$ in $P_{\alpha}$ is annihilated by $\delta_{i}$.

Since the arrangement defined by $p_{1}, \ldots, p_{r}$ is generic, each $\delta_{i}$ annihilates precisely $n-1$ of the $p_{j}$ :s. Thus, for every $i=1, \ldots, s$, we have $\operatorname{deg} P_{i}=r-n+1$. Lemma 5.34 shows that we may write $P_{\alpha} \delta^{\alpha}=P^{\prime} P_{i} \delta_{i}^{\alpha_{i}} \delta^{\alpha-\alpha_{i} e_{i}}$ as a sum in which each term is of the form

$$
a P^{\prime} P_{i} \delta_{i}^{m_{1}} \cdots P_{i} \delta_{i}^{m_{l}} \delta^{\alpha-\alpha_{i} e_{i}},
$$

where $a \in k \backslash\{0\}$ and $1 \leq m_{j} \leq 2(r-n)+3$, for $j=1, \ldots, l$.

Since $\delta_{i}$ annihilates all factors in $P^{\prime}$, it annihilates $P^{\prime}$. Whence $P^{\prime}$ and $\delta_{i}$ commute, so

$$
\begin{aligned}
a P^{\prime} P_{i} \delta_{i}^{m_{1}} \cdots P_{i} \delta_{i}^{m_{l}} \delta^{\alpha-\alpha_{i} e_{i}} & =a P_{i} \delta_{i}^{m_{1}} \cdots P_{i} \delta_{i}^{m_{l-1}} P^{\prime} P_{i} \delta_{i}^{m_{l}} \delta^{\alpha-\alpha_{i} e_{i}} \\
& =a P_{i} \delta_{i}^{m_{1}} \cdots P_{i} \delta_{i}^{m_{l-1}} P_{\alpha} \delta_{1}^{\alpha_{1}} \cdots \delta_{i}^{m_{l}} \cdots \delta_{s}^{\alpha_{s}} \\
& =a P_{i} \delta_{i}^{m_{1}} \cdots P_{i} \delta_{i}^{m_{l-1}} P_{\nu} \delta^{\nu}
\end{aligned}
$$

where $\nu=\left(\alpha_{1}, \ldots, \alpha_{i-1}, m_{l}, \alpha_{i+1}, \ldots, \alpha_{s}\right)$, and $P_{\nu}=P_{\alpha}$, since $m_{l} \geq 1$.

We may now do the same thing with $P_{\nu} \delta^{\nu}$, if some $\nu_{j}>2(r-n)+3$, and continuing like this, we will eventually end up with a $k$-linear combination of products of operators of the form $P_{\beta} \delta^{\beta}$ with $\beta_{i} \leq 2(r-n)+3$, for each $i=1, \ldots, s$, and such that $\beta_{i} \neq 0$ only if $\alpha_{i} \neq 0$.

Hence $\left\{x_{1}, \ldots, x_{n}, \varepsilon\right\} \cup\left\{P_{\alpha} \delta^{\alpha} \mid \alpha_{i} \leq 2(r-n)+3\right.$ for $\left.i=1, \ldots, s\right\}$ generate $\mathcal{D}(I)$ as a $k$-algebra.

By applying Theorem 2.5 we get

Corollary 5.36. Let $A$ be the coordinate ring of a generic arrangement, defined by the ideal $\left\langle p_{1} \cdots p_{r}\right\rangle \subseteq R$. Then $\mathcal{D}(A)$ is finitely generated as a $k$-algebra.

With notation as in Theorem 5.35:

$$
\text { If } r \leq n \text {, then } \mathcal{D}(A) \text { is generated by }
$$

$$
\left\{\overline{x_{1}}, \ldots, \overline{x_{n}}, \delta_{r+1}, \ldots, \delta_{n}\right\} \cup\left\{\overline{p_{i}} \delta_{i}, \overline{p_{i}} \delta_{i}^{2}, \overline{p_{i}} \delta_{i}^{3} \mid i=1, \ldots, r\right\} .
$$




$$
\begin{aligned}
& \text { If } r>n \text {, then } \mathcal{D}(A) \text { is generated by } \\
& \qquad\left\{\overline{x_{1}}, \ldots, \overline{x_{n}}, \bar{\varepsilon}\right\} \cup\left\{\overline{P_{\alpha}} \delta^{\alpha} \mid \alpha_{i} \leq 2(r-n)+3 \text { for } i=1, \ldots, s\right\}, \\
& \text { where } \bar{\varepsilon}=\sum \overline{x_{i}} \partial_{i} .
\end{aligned}
$$

Remark. As we remarked after Corollary 5.29, $\overline{P_{\alpha}} \neq 0$ if and only if $\delta^{\alpha} \in V_{i}^{|\alpha|}$, for some $i$.

Again, the case $n=2$ takes a special position. Before stating our next proposition we will need a definition to facilitate its proof.

Definition 5.37. For a monomial $x^{\alpha} \partial^{\beta} \in \mathcal{D}(R)$, we define its total degree, as totdeg $\left(x^{\alpha} \partial^{\beta}\right)=|\alpha|-|\beta|$. A nonzero operator $\theta$ is of homogeneous total degree $t$, if all monomials occuring in $\theta$ have total degree $t$. In this case we write $t=\operatorname{totdeg}(\theta)$.

It is clear that if $\theta$ and $\psi$ are operators of homogeneous total degree, then so is $\theta \psi$, and

$$
\operatorname{totdeg}(\theta \psi)=\operatorname{totdeg}(\psi \theta)=\operatorname{totdeg}(\theta)+\operatorname{totdeg}(\psi) .
$$

Proposition 5.38. Suppose $p_{1}, \ldots, p_{r} \subseteq k[x, y]$ define a central arrangement. Let $P_{i}$ and $\delta_{i}$ be as in Corollary 5.30, and let $A$ be the coordinate ring of the arrangement. Then $\mathcal{D}(A)$ is generated, as a $k$-algebra, by

$$
\left\{\bar{y}, \delta_{1}\right\} \quad \text { or } \quad\{\bar{x}, \bar{y}\} \cup\left\{\overline{p_{1}} \delta_{1}^{m}, \overline{p_{2}} \delta_{2}^{m} \mid m=1,2,3\right\},
$$

if $r$ is one or two, respectively, and by

$$
\{\bar{x}, \bar{y}, \bar{\varepsilon}\} \cup\left\{\bar{P}_{i} \delta_{i}^{m} \mid i=1, \ldots, r \text { and } m=1, \ldots, 2 r-1\right\},
$$

if $r \geq 3$. Thus $\mathcal{D}(A)$ is generated by operators of order $\leq 2 r-1$. Moreover, $\mathcal{D}(A)$ cannot be generated by operators of lower order than this.

Proof. The first part of the proposition follows from Corollary 5.36 together with the subsequent remark.

To show the second part, let $I$ be the defining ideal for the arrangement under consideration. By Corollary 5.30, any product of $k$-algebra generators of $\mathcal{D}(I)$ is a $k$-linear combination of products of $x, y, \varepsilon$, and operators of the form $P_{i} \delta_{i}^{m}$ for some $m$, together with operators with polynomial coefficients in $I$. All operators of the form $P_{i} \delta_{i}^{m}$, as well as $\varepsilon$, have homogeneous total degree. Since $\operatorname{totdeg}(x)=\operatorname{totdeg}(y)=1$ and $\operatorname{totdeg}(\varepsilon)=0$, if $\theta$ is any product of $k$-algebra generators for $\mathcal{D}(I)$, not inducing the zero operator in $\mathcal{D}(A)$, it must be a sum of operators $\psi$ of homogeneous total degree, such that

$$
\operatorname{totdeg}(\psi) \geq \operatorname{totdeg}\left(P_{i} \delta_{i}^{m_{1}} \cdots P_{i} \delta_{i}^{m_{t}}\right)=t(r-1)-\sum_{l=1}^{t} m_{l},
$$

for some $i$. Assuming that $m_{l}<2 r-1$ for each $l$ we get

$$
\begin{aligned}
\operatorname{totdeg}\left(P_{i} \delta_{i}^{m_{1}} \cdots P_{i} \delta_{i}^{m_{t}}\right) & =t(r-1)-\sum_{l=1}^{t} m_{l} \geq t(r-1)-2 t(r-1) \\
& =t(1-r)>-r=\operatorname{totdeg}\left(P_{j} \delta_{j}^{2 r-1}\right),
\end{aligned}
$$

for each $j$. Hence no $\bar{P}_{j} \delta_{j}^{2 r-1}$ can be expressed using lower order operators in $\mathcal{D}(A)$. 


\section{References}

[1] J.-E. Björk, Rings of Differential Operators, North-Holland Mathematical Library 21, Amsterdam, 1979.

[2] G. Másson, Two-sided ideals in Rings of Differential Operators and Étale Homomorphisms, J. Algebra 171 (1995), 703-712.

[3] J.C. McConnell and J.C. Robson, Noncommutative Noetherian Rings, Pure and Applied Mathematics, John Wiley \& Sons, Chichester, 1987.

[4] P. Orlik and H. Terao, Arrangements of Hyperplanes, Grundlehren der matematischen Wissenschaften 300, Springer-Verlag, 1992.

[5] B. Singh, Differential Operators on a Hypersurface, Nagoya Math. J. 103 (1986), 67-84.

[6] W. Traves, Nakai's conjecture for varieties smoothed by normalization, Proc. Amer. Math. Soc. 127 (8) (1999), 2245-2248.

[7] J. Wiens, The Module of Derivations for an Arrangement of Subspaces, Pacific J. Math. 198 (2001), 501-512.

[8] A. Zaharia, On Hilbert Polynomials of Modules of Logarithmic Vector Fields of Arrangements, Comm. Math. Univ. Sancti Pauli 48 (1999), 717.

Pär Holm

pho@matematik.su.se

Department of Mathematics

Stockholm University

SE-106 91 Stockholm

Sweden 